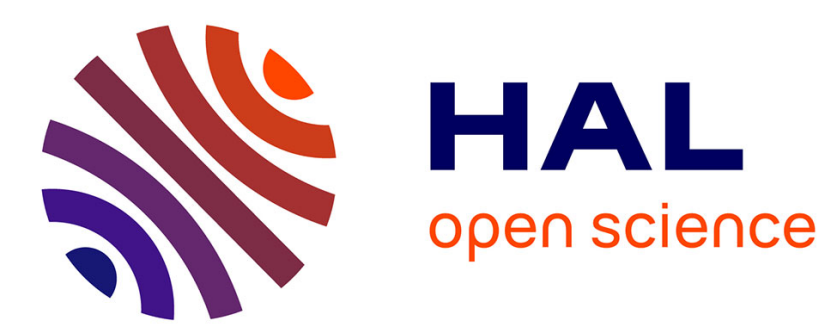

\title{
Hermite Subdivision Schemes and Taylor Polynomials
}

Serge Dubuc, Jean-Louis Merrien

\section{To cite this version:}

Serge Dubuc, Jean-Louis Merrien. Hermite Subdivision Schemes and Taylor Polynomials. Constructive Approximation, 2009, 29 (2), pp.219-245. 10.1007/s00365-008-9011-5 . hal-00133615

\section{HAL Id: hal-00133615 https://hal.science/hal-00133615}

Submitted on 27 Feb 2007

HAL is a multi-disciplinary open access archive for the deposit and dissemination of scientific research documents, whether they are published or not. The documents may come from teaching and research institutions in France or abroad, or from public or private research centers.
L'archive ouverte pluridisciplinaire HAL, est destinée au dépôt et à la diffusion de documents scientifiques de niveau recherche, publiés ou non, émanant des établissements d'enseignement et de recherche français ou étrangers, des laboratoires publics ou privés. 


\title{
Hermite Subdivision Schemes and Taylor Polynomials
}

\author{
Serge Dubuc and Jean-Louis Merrien
}

February 20, 2007

\begin{abstract}
We propose a general study of the convergence of a Hermite subdivision scheme $\mathcal{H}$ of degree $d>0$ in dimension 1 . This is done by linking Hermite subdivision schemes and Taylor polynomials and by associating a so-called Taylor subdivision (vector) scheme $\mathcal{S}$. The main point of investigation is a spectral condition. If the subdivision scheme of the finite differences of $\mathcal{S}$ is contractive, then $\mathcal{S}$ is $C^{0}$ and $\mathcal{H}$ is $C^{d}$. We apply this result to two families of Hermite subdivision schemes, the first one is interpolatory, the second one is a kind of corner cutting, both of them use Obreshkov interpolation polynomial.
\end{abstract}

\section{Introduction}

A Hermite subdivision scheme $\mathcal{H}$ of degree $d$ is a recursive scheme for computing a function $\phi: \mathbb{R} \rightarrow \mathbb{R}$ and its $d$ derivatives $\phi^{\prime}, \ldots, \phi^{(d)}$. The initial state of the scheme is a vector function $f_{0}: \mathbb{Z} \rightarrow \mathbb{R}^{d+1}$. The first component of $f_{0}$ is a control value for $\phi$, the second component, for $\phi^{\prime}$ and so on. The sequence of refinements $f_{n}: \mathbb{Z} \rightarrow \mathbb{R}^{d+1}, n>0$, is recursively defined through a family of $(d+1) \times(d+1)$ matrices $\left\{A(\alpha)=\left(a_{i j}(\alpha)\right)_{i, j=0, \ldots, d}\right\}_{\alpha \in \mathbb{Z}}$, a finite number of them being non-zero, by

$$
D^{n+1} f_{n+1}(\alpha)=\sum_{\beta \in \mathbb{Z}} A(\alpha-2 \beta) D^{n} f_{n}(\beta), \alpha \in \mathbb{Z}, n \geq 0
$$

where $D$ is the diagonal matrix whose diagonal elements are $1,1 / 2, \ldots, 1 / 2^{d}$.

Another way of writing the previous equation is

$$
f_{n+1}^{(i)}(\alpha) / 2^{i(n+1)}=\sum_{\beta \in \mathbb{Z}} \sum_{j=0}^{d} a_{i j}(\alpha-2 \beta) f_{n}^{(j)}(\beta) / 2^{j n}, i=0,1, \ldots, d
$$


for $\alpha \in \mathbb{Z}$, where $f_{n}(\alpha)=\left(f_{n}^{(0)}(\alpha), \ldots, f_{n}^{(d)}(\alpha)\right)^{T} \in \mathbb{R}^{d+1}$.

The family of matrices $\{A(\alpha)\}_{\alpha \in \mathbb{Z}}$ is called the mask of the Hermite subdivision scheme $\mathcal{H}$. The support of $\mathcal{H}$ is the smallest interval $\left[\sigma, \sigma^{\prime}\right]$ containing $\{\alpha \in \mathbb{Z}: A(\alpha) \neq$ $0\}$.

The analysis of interpolatory Hermite subdivision schemes of degree 1 has been initiated by Merrien [13] and by Dyn and Levin [7]. The first investigations of Hermitetype subdivision schemes of degree larger than 1 have been proposed by Merrien [14], Dyn and Levin [8], Zhou [18], Han [9] and Yu [17]. They have given the theory and the tools (through Fourier transforms for the last three) for analyzing the convergence and smoothness of Hermite-type interpolatory schemes. However, there has been no such analysis for noninterpolatory Hermite subdivision schemes. In this paper, we expect to cover this gap.

The paper is divided into seven sections including the introduction. In Section 2, we define the notion of $C^{d}$ convergence for a Hermite subdivision scheme $\mathcal{H}$ of degree $d$ and the Taylor condition about the refinements of $\mathcal{H}$. The main result of Section 3 is that a nondegenerate Hermite subdivision scheme $\mathcal{H}$ satisfying a weak form of the Taylor condition always satifies a spectral condition. This spectral condition has many implications such as the reproduction of polynomials. In Section 4, with every Hermite subdivision scheme $\mathcal{H}$ satisfying the spectral condition, we associate a vector subdivision scheme $\mathcal{S}$, the so-called Taylor subdivision scheme. In Section 5 , we propose a sufficient condition for $C^{d}$ convergence of a Hermite subdivision scheme of degree $d$. This criterion is that the subdivision matrix $T$ of the finite differences arising from the Taylor subdivision scheme is contractive. When this is the case, the Taylor subdivision scheme is $C^{0}$ and the Hermite subdivision scheme is $C^{d}$. In Section 6 , for every positive integer $d$, we consider two specific Hermite subdivision schemes $H I S^{d}$ and $H C C^{d}$. The first one is interpolatory by solving a basic Hermite problem whose solution is a piecewise Hermite polynomial. The second one is non interpolatory and is a kind of corner cutting which is a generalization of the well known Chaikin's algorithm. For each of these schemes, for $d=1,2,3$, we compute the subdivision matrix $T$ and we check that its spectral radius is $<1$.

\section{Convergence and Taylor Condition}

We define the notion of $C^{d}$ convergence for a Hermite subdivision scheme $\mathcal{H}$ of degree $d$ and the Taylor condition about the refinements of $\mathcal{H}$.

Definition 1 We say that a Hermite subdivision scheme of degree $d$ is $C^{d}$ if for every sequence of refinements $f_{n}: \mathbb{Z} \rightarrow \mathbb{R}^{d+1}$, there exists a $C^{d}$-function $\phi: \mathbb{R} \rightarrow \mathbb{R}$ for which for every $\epsilon>0$ and for every $L>0$, there exists $N$ such that for every $n>N$, for every $\alpha \in\left[-L 2^{n}, L 2^{n}\right],\left|f_{n}^{(0)}(\alpha)-\phi\left(\alpha / 2^{n}\right)\right|<\epsilon,\left|f_{n}^{(1)}(\alpha)-\phi^{\prime}\left(\alpha / 2^{n}\right)\right|<\epsilon$, 
$\ldots,\left|f_{n}^{(d)}(\alpha)-\phi^{(d)}\left(\alpha / 2^{n}\right)\right|<\epsilon$. The function $\phi$ is called the limit function associated to the refinements $f_{n}$.

Definition 2 Let $d$ be a positive integer, we say that a sequence of $f_{n}: \mathbb{Z} \rightarrow \mathbb{R}^{d+1}$ fulfills the Taylor condition if for every $\epsilon>0$ and for every $L>0$, there exists $N$ such that for every $n>N$,

$$
\left|f_{n}^{(i)}(\alpha+1) / 2^{i n}-\sum_{j=i}^{d} \frac{1}{(j-i) !} f_{n}^{(j)}(\alpha) / 2^{j n}\right|<\epsilon / 2^{d n}
$$

for $i=0, \ldots, d$, for every $\alpha \in\left[-L 2^{n}, L 2^{n}\right]$. The Taylor condition is satisfied by a Hermite subdivision scheme if every sequence of its refinements fulfills the Taylor condition.

Lemma 1 Let $\phi: \mathbb{R} \rightarrow \mathbb{R}$ be a $C^{d}$ function. If for every $\alpha \in \mathbb{Z}$, we set $f_{n}^{(0)}(\alpha)=$ $\phi\left(\alpha / 2^{n}\right), f_{n}^{(1)}(\alpha)=\phi^{\prime}\left(\alpha / 2^{n}\right), \ldots, f_{n}^{(d)}(\alpha)=\phi^{(d)}\left(\alpha / 2^{n}\right)$, then the Taylor condition (3) is satisfied by the sequence $f_{n}=\left(f_{n}^{(0)}, f_{n}^{(1)}, \ldots, f_{n}^{(d)}\right)^{T}$.

Proof: Let $i \in\{0,1, \ldots, d\}$, the Taylor expansion gives the existence of $\left.\theta_{i} \in\right] 0,1[$ such that

$$
\phi^{(i)}\left((\alpha+1) / 2^{n}\right)=\sum_{j=i}^{d-1} \frac{\phi^{(j)}\left(\alpha / 2^{n}\right)}{(j-i) !} / 2^{n(j-i)}+\frac{\phi^{(d)}\left(\left(\alpha+\theta_{i}\right) / 2^{n}\right)}{(d-i) !} / 2^{n(d-i)}
$$

or equivalently

$$
\frac{\phi^{(i)}\left((\alpha+1) / 2^{n}\right)}{2^{i n}}-\sum_{j=i}^{d} \frac{\phi^{(j)}\left(\alpha / 2^{n}\right)}{(j-i) ! 2^{j n}}=\frac{\phi^{(d)}\left(\left(\alpha+\theta_{i}\right) / 2^{n}\right)-\phi^{(d)}\left(\alpha / 2^{n}\right)}{(d-i) ! 2^{d n}}
$$

From that, for every $L>0$, since $\phi^{(d)}$ is uniformly continuous on $[-L, L+1]$, for $i=0, \ldots, d$,

$$
\phi^{(i)}\left((\alpha+1) / 2^{n}\right) / 2^{i n}-\sum_{j=i}^{d} \frac{1}{(j-i) !} \phi^{(j)}\left(\alpha / 2^{n}\right) / 2^{j n}=o\left(1 / 2^{d n}\right)
$$

uniformly for every $\alpha \in\left[-L 2^{n}, L 2^{n}\right]$ as $n \rightarrow \infty$.

A Hermite subdivision scheme is interpolatory if $A(0)=D$ and for all $\alpha \in \mathbb{Z}$ with $\alpha \neq 0, A(2 \alpha)=0$. In this case, for $\alpha \in \mathbb{Z}, f_{n}(\alpha)=f_{n+1}(2 \alpha)$.

Corollary 2 In a given interpolatory $C^{d}$ Hermite subdivision scheme of degree $d$, the Taylor condition (3) is satisfied. 


\section{Spectral properties of Hermite schemes}

The main result of this section is that a nondegenerate Hermite subdivision scheme $\mathcal{H}$ satisfying a weak form of the Taylor condition will verify a spectral condition. This spectral condition has many implications, in the first instance the determination of the behavior of the refinements of $\mathcal{H}$. Before reaching the main result, some lemmas are required. We use the notation $\mathcal{P}_{d}$ for the space of polynomials of degree inferior or equal to $d$.

Lemma 3 Let $p(x) \in \mathcal{P}_{d}$ and $f(\alpha)=\left(p(\alpha), p^{\prime}(\alpha), \ldots, p^{(d)}(\alpha)\right)^{T}$, for $\alpha \in \mathbb{Z}$, then

$$
f^{(i)}(\alpha+1)-\sum_{j=i}^{d} f^{(j)}(\alpha) /(j-i) !=0
$$

for $i=0,1, \ldots, d$, for every $\alpha \in \mathbb{Z}$.

Proof: Let $p(x) \in \mathcal{P}_{d}$, then $p^{(d+1)}(x)=0$. Let $i \in\{0,1, \ldots, d\}$, the Taylor expansion of $p^{(i)}$ (the $i$ th derivative of $p$ ) is $p^{(i)}(x)=\sum_{j=i}^{d} p^{(j)}(a)(x-a)^{j-i} /(j-i)$ !. We set $a=\alpha, x=\alpha+1$, we obtain $f^{(i)}(\alpha+1)=\sum_{j=i}^{d} f^{(j)}(\alpha) /(j-i)$ !.

Lemma 4 Let $f=\left(f^{(0)}, f^{(1)}, \ldots, f^{(d)}\right)^{T}$ where $f^{(i)}:[a, b] \cap \mathbb{Z} \rightarrow \mathbb{R}, i=0,1, \ldots, d$ and where $b-a \geq 1$, we assume that for $i=0,1, \ldots, d$, for every $\alpha \in[a, b-1] \cap \mathbb{Z}$,

$$
f^{(i)}(\alpha+1)-\sum_{j=i}^{d} f^{(j)}(\alpha) /(j-i) !=0,
$$

then there exists a polynomial $p$ of degree less than or equal to $d$ such that $f(\alpha)=$ $\left(p(\alpha), p^{\prime}(\alpha), \ldots, p^{(d)}(\alpha)\right)^{T}$ for every $\alpha \in[a, b] \cap \mathbb{Z}$.

Proof: If $a \notin \mathbb{Z}$, we substitute $a$ by $\min [a, b] \cap \mathbb{Z}$ and we set $p(x)=\sum_{k=0}^{d} f^{(k)}(a) / k !(x-$ $a)^{k}$ and $v(\alpha)=\left(p(\alpha), p^{\prime}(\alpha), \ldots, p^{(d)}(\alpha)\right)^{T}$ and $h(\alpha)=f(\alpha)-v(\alpha)$ for $\alpha \in[a, b] \cap \mathbb{Z}$. We define the linear map $Q f=g$ where $g:[a, b-1] \cap \mathbb{Z} \rightarrow \mathbb{R}^{d+1}$ is defined as follows

$$
g^{(i)}(\alpha)=f^{(i)}(\alpha+1)-\sum_{j=i}^{d} f^{(j)}(\alpha) /(j-i) !, \quad i=0,1, \ldots, d .
$$

From Lemma 3, $Q v=0$. By hypothesis, $Q f=0$, thus $Q h=0$. It follows that for every $\alpha \in[a, b-1]$, and for $i=0,1, \ldots, d$, we get $h^{(i)}(\alpha+1)=\sum_{j=i}^{d} h^{(j)}(\alpha) /(j-i)$ !. Since $h(a)=0$, by induction, we get that for every $\alpha \in[a, b], h(\alpha)=0$ so that $f=v$.

The two previous lemmas provide a characterization of the finite difference equation (4). We recall a lemma (Proof can be found in [5] Lemma 20) 
Lemma 5 Let $z_{0}, z_{2}, \ldots, z_{d}$ be $d+1$ distinct complex numbers and $p_{0}, p_{1}, \ldots, p_{d}$ be $d+1$ nonzero vector polynomials with values in the same finite vector space, we assume that the sequence of vectors $\sum_{k=0}^{d} p_{k}(n) z_{k}^{n}$ converges to $c$. If $c \neq 0$, then there exists an integer $j \in[0, d]$ such that $z_{j}=1$ and $p_{j}(n) \equiv c$ and for every other integer $k \in[0, d]$, $\left|z_{k}\right|<1$. If $c=0$, then for every integer $k \in[0, d],\left|z_{k}\right|<1$.

Lemma 6 Let $\mathcal{H}$ be a Hermite subdivision scheme of degree $d$ with mask $\{A(\alpha)\}_{\alpha \in \mathbb{Z}}$ whose support is contained in $\left[\sigma, \sigma^{\prime}\right]$. Let $E$ be the set of vectors $v=\left(v^{(0)}, v^{(1)}, \ldots, v^{(d)}\right)^{T}$ where $v^{(i)}:\left[-\sigma^{\prime},-\sigma\right] \rightarrow \mathbb{C}, i=0,1, \ldots, d$. We consider the linear operator $T$ in $E$ : $T v(\alpha)=\sum_{\beta=-\sigma^{\prime}}^{-\sigma} A(\alpha-2 \beta) v(\beta)$. Let $v \in E$ and let $f_{n}$ be a sequence of refinements $f_{n}$ such that $\forall \alpha \in\left[-\sigma^{\prime},-\sigma\right] f_{0}(\alpha)=v(\alpha)$, then $\forall \alpha \in\left[-\sigma^{\prime},-\sigma\right] D^{n} f_{n}(\alpha)=T^{n} v(\alpha)$.

Proof: We set $v_{n}=T^{n} v$ for $n=0,1,2, \ldots$. We prove by induction that $\forall \alpha \in$ $\left[-\sigma^{\prime},-\sigma\right] D^{n} f_{n}(\alpha)=v_{n}(\alpha)$. By hypothesis, this is the case for $n=0$. Let us assume that for a given integer $n, \forall \alpha \in\left[-\sigma^{\prime},-\sigma\right] D^{n} f_{n}(\alpha)=v_{n}(\alpha)$. If $\alpha \in \mathbb{Z}$, then we obtain

$$
D^{n+1} f_{n+1}(\alpha)=\sum_{\beta \in \mathbb{Z}} A(\alpha-2 \beta) D^{n} f_{n}(\beta) .
$$

If $\alpha \in\left[-\sigma^{\prime},-\sigma\right]$ and $\beta \notin\left[-\sigma^{\prime},-\sigma\right]$, then $\alpha-2 \beta \notin\left[-\sigma^{\prime},-\sigma\right]$ and $A(\alpha-2 \beta)=0$. It follows that

$$
D^{n+1} f_{n+1}(\alpha)=\sum_{\beta=-\sigma^{\prime}}^{-\sigma} A(\alpha-2 \beta) D^{n} f_{n}(\beta)=T v_{n}(\alpha)=v_{n+1}(\alpha)
$$

whenever $\alpha \in\left[-\sigma^{\prime},-\sigma\right]$.

Definition 3 A Hermite subdivision scheme of degree $d$ is nondegenerate if for each $i \in\{0,1, \ldots, d\}$, there exists an integer $a_{i}$ and a sequence $f_{n}$ of refinements such that $\lim _{n \rightarrow \infty} f_{n}^{(i)}\left(a_{i}\right) \neq 0$

Theorem 7 Let $\mathcal{H}$ be a nondegenerate Hermite subdivision scheme of degree $d$ with

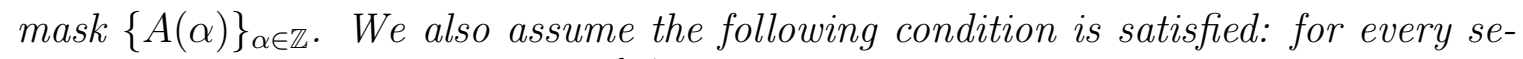
quence of refinements $f_{n}: \mathbb{Z} \rightarrow \mathbb{R}^{d+1}$,

$$
f_{n}^{(i)}(\alpha+1) / 2^{i n}-\sum_{j=i}^{d} \frac{1}{(j-i) !} f_{n}^{(j)}(\alpha) / 2^{j n}=o\left(1 / 2^{d n}\right) \quad \alpha \in \mathbb{Z}, i=0,1, \ldots, d .
$$

Then for every $k=0, \ldots, d$, there is a unique polynomial $p_{k}(x)$ of degree $k$ with its leading coefficient equal to $1 / k$ ! such that the vector function $v_{k}: \mathbb{Z} \rightarrow \mathbb{R}^{d+1}$ where $v_{k}(\alpha)=\left(p_{k}(\alpha), \ldots, p_{k}^{(d)}(\alpha)\right)^{T}$ is an eigenvector of $(A(\alpha-2 \beta))_{\alpha, \beta \in \mathbb{Z}}$ for the eigenvalue 
$1 / 2^{k}$. Moreover, if $f_{n}$ is a sequence of refinements of $\mathcal{H}$, then there exist constants $c_{0}, c_{1}, \ldots, c_{d}$ such that for every $\alpha \in \mathbb{Z}$,

$$
f_{n}^{(i)}(\alpha) / 2^{i n}=\sum_{k=0}^{d} c_{k} p_{k}^{(i)}(\alpha) / 2^{k n}+o\left(1 / 2^{d n}\right), \quad i=0,1, \ldots, d .
$$

In particular, $\lim _{n \rightarrow \infty} f_{n}^{(i)}(\alpha)=c_{i}$ for $i=0,1, \ldots, d$.

Proof: For $i \in\{0, \ldots, d\}$, let $f_{n}$ be a sequence of refinements and $a_{i} \in \mathbb{Z}$ such that

$$
\lim _{n \rightarrow \infty} f_{n}^{(i)}\left(a_{i}\right)=1
$$

$(\mathcal{H}$ is nondegenerate).

Let $\sigma, \sigma^{\prime} \in \mathbb{Z}$ be such that the support of $\mathcal{H}$ is contained in $\left[\sigma, \sigma^{\prime}\right]$ and $a_{i} \in$ $\left[-\sigma,-\sigma^{\prime}\right]$. If $E$ is the set of vectors $v=\left(v^{(0)}, v^{(1)}, \ldots, v^{(d)}\right)^{T}$ where $v^{(i)}:\left[-\sigma^{\prime},-\sigma\right] \rightarrow \mathbb{C}$, $i=0,1, \ldots, d$, we define the linear operator $T$ in $E$ by $T v(\alpha)=\sum_{\beta=-\sigma^{\prime}}^{-\sigma} A(\alpha-2 \beta) v(\beta)$. Let us denote $m(z)=\prod_{k=1}^{K}\left(z-\lambda_{k}\right)^{\mu_{k}}$ the minimal polynomial of $T$ where the $\lambda_{k}$ are distinct, then we define $E_{k}$ as the kernel of $\left(T-\lambda_{k} I\right)^{\mu_{k}}$. According to the primary decomposition of a vector space (see Theorem 4.2 in Lang [10]), $E$ is the direct sum of $E_{k}: E=\oplus_{k=1}^{K} E_{k}$.

Let $F$ be the set of vectors $w=\left(w^{(0)}, w^{(1)}, \ldots, w^{(d)}\right)^{T}$ for which there exists a polynomial $p \in \mathcal{P}_{d}$ satisfying for $i=0, \ldots, d$, for all $\alpha \in\left[-\sigma^{\prime},-\sigma\right] w^{(i)}(\alpha)=p^{(i)}(\alpha)$. If $\lambda_{k}$ is an eigenvalue such that $\left|\lambda_{k}\right| \geq 1 / 2^{d}$, let us show that $E_{k} \subset F$.

For $v \in E_{k}$, we define the sequence: $v_{0}=v, v_{\ell+1}=\left(T-\lambda_{k} I\right) v_{l}, \ell \geq 0$ and finally we set $\nu=\min \left\{\ell \geq 0: v_{\ell+1}=0\right\}$. Let $f_{0}: \mathbb{Z} \rightarrow \mathbb{R}^{d+1}$ be defined by $\forall \alpha \in$ $\left[-\sigma^{\prime},-\sigma\right], f_{0}(\alpha)=v(\alpha)$ (for any $\alpha \notin\left[-\sigma^{\prime},-\sigma\right]$, the choice of $f_{0}(\alpha)$ may be arbitrary), we consider the sequence of refinements generated by $f_{0}$. By Lemma 6 , we obtain $D^{n} f_{n}(\alpha)=T^{n} v(\alpha)$ for $n=0,, 1,2, \ldots$ and $\forall \alpha \in\left[-\sigma^{\prime},-\sigma\right]$. Since $T v_{\ell}=v_{\ell+1}+\lambda_{k} v_{\ell}$ and $v_{\ell}=0$ for $\ell>\nu$, we can prove recursively that $T^{n} v=\sum_{\ell=0}^{\nu}\left(\begin{array}{l}n \\ \ell\end{array}\right) \lambda_{k}^{n-\ell} v_{\ell}$ with $\left(\begin{array}{l}n \\ \ell\end{array}\right)=0$ if $n<\ell$. This can be written

$$
f_{n}^{(i)}(\alpha) / 2^{i n}=\sum_{\ell=0}^{\nu}\left(\begin{array}{l}
n \\
\ell
\end{array}\right) \lambda_{k}^{n-\ell} v_{\ell}^{(i)}(\alpha) \quad i=0, \ldots, d, \alpha \in\left[-\sigma^{\prime},-\sigma\right]
$$

From (5) and (8), we obtain

$$
\lim _{n \rightarrow \infty} \sum_{\ell=0}^{\nu}\left(\begin{array}{l}
n \\
\ell
\end{array}\right) 2^{d n} \lambda_{k}^{n-\ell}\left[v_{\ell}^{(i)}(\alpha+1)-\sum_{j=i}^{d} \frac{v_{\ell}^{(j)}(\alpha)}{(j-i) !}\right]=0
$$

for any $\alpha \in\left[-\sigma^{\prime},-\sigma-1\right]$, and $i=0,1, \ldots, d$. From (9) and Lemma 5 , since $2^{d}\left|\lambda_{k}\right| \geq 1$, we get $v^{(i)}(\alpha+1)-\sum_{j=i}^{d} \frac{v^{(j)}(\alpha)}{(j-i) !}=0$. From Lemma 4 , there exists a polynomial $p_{k} \in \mathcal{P}_{d}$ 
such that $v^{(0)}(\alpha)=p_{k}(\alpha), v^{(1)}(\alpha)=p_{k}^{\prime}(\alpha), \ldots, v^{(d)}(\alpha)=p_{k}^{(d)}(\alpha), \alpha \in\left[-\sigma^{\prime},-\sigma\right]$. Thus $E_{k} \subset F$. From this inclusion of sets, we obtain the inequality

$$
\sum_{\left|\lambda_{k}\right| \geq 1 / 2^{d}} \operatorname{dim} E_{k} \leq \operatorname{dim} F=d+1
$$

Let $i \in\{0, \ldots, d\}, a_{i} \in\left[-\sigma^{\prime},-\sigma\right]$ and let $f_{n}$ be a sequence of refinements such that (7) is satisfied. We set $w(\alpha)=f_{0}(\alpha), \alpha \in\left[-\sigma^{\prime},-\sigma\right]$. The vector $w$ has a unique decomposition in $E: w=\sum_{k=0}^{K} w_{k}$ with $w_{k} \in E_{k}$. For $k=1, \ldots, K$, we define the sequence: $w_{k 0}=w_{k}, w_{k, \ell+1}=\left(T-\lambda_{k} I\right) w_{k l}, \ell \geq 0$ and finally we set $\nu_{k}=\min \left\{\ell \geq 0: w_{k, \ell+1}=0\right\}$. Then $T^{n} w=\sum_{k=0}^{K} \sum_{\ell=0}^{\nu_{k}}\left(\begin{array}{l}n \\ \ell\end{array}\right) w_{k \ell} \lambda_{k}^{n-\ell}$. More precisely, we get

$$
f_{n}^{(i)}(\alpha) / 2^{i n}=\sum_{k=0}^{K} \sum_{\ell=0}^{\nu_{k}}\left(\begin{array}{l}
n \\
\ell
\end{array}\right) \lambda_{k}^{n-\ell} w_{k \ell}^{(i)}(\alpha), \quad \forall \alpha \in\left[-\sigma^{\prime},-\sigma\right], i=0,1, \ldots, d .
$$

From (7), (11) and from Lemma 5, we deduce that $1 / 2^{i}$ is an eigenvalue of $T$.

Since this is true for $i=0, \ldots, d$, from (10), we obtain that $\operatorname{dim} E_{k}=1$ if $\lambda_{k}=$ $1 / 2^{i}$, this means that $\nu_{k}=0$. The eigenvector corresponding to eigenvalue $\lambda_{k}$ is the vector $v_{k}=\left(p_{k}, p_{k}^{\prime}, \ldots, p_{k}^{(d)}\right)^{T}$. Any eigenvalue not in $\left\{1 / 2^{i}: i=0,1, \ldots, d\right\}$ is in the disk $|\lambda|<1 / 2^{d}$. If we reorder the eigenvalues in such a way that $\lambda_{k}=1 / 2^{k}$ for $k=0,1, \ldots d$, then Equation (11) becomes Equation (6) where $c_{k}$ is the number defined by $w_{k}=c_{k} v_{k}$.

Let us show that the degree of $p_{k}$ is $k$ for $k=0,1, \ldots, d$. In (6) let us use the sequence of refinements $f_{n}$ for which (7) is satisfied. From Lemma 5 , we get $c_{i} p_{i}^{(i)}\left(a_{i}\right) \neq$ 0 and $c_{j} p_{j}^{(i)}\left(a_{i}\right)=0$ for for $i=0,1, \ldots, d$ and $j<i$. We obtain that $c_{i} \neq 0$ for $i=0,1, \ldots, d, p_{i}^{(i)}\left(a_{i}\right) \neq 0$ and $p_{j}^{(i)}\left(a_{i}\right)=0$ for $j<i$. The degree of $p_{k}$ is $\max \{\ell$ : $\left.p_{k}^{\ell}\left(a_{i}\right) \neq 0\right\}=k$ since we know that $p_{k}^{(\ell)}\left(a_{i}\right)=0$ for $k<\ell$.

Since the degree of $p_{k}$ is $k$, then for $i, k \in\{0,1, \ldots, d\}$, the limit of $p_{k}^{(i)}(\alpha) 2^{(i-k) n}$ is $\delta_{i k}$ and the limit of $f_{n}^{(i)}(\alpha)$ is $c_{i}$ as $n \rightarrow \infty$.

As $\sigma$ and $\sigma^{\prime}$ may be chosen arbitrarily large in absolute values and that the polynomials $p_{k}$ are unique up to a multiplicative factor, the proof is complete.

Remark 1 If the Taylor condition is satisfied by a sequence of vector function $f_{n}$ : $\mathbb{Z} \rightarrow \mathbb{R}^{d+1}$, then its weak form (5) is valid.

We put the main statement of the previous theorem in the form of a definition.

Definition 4 A Hermite subdivision scheme of degree d satisfies the spectral condition if for $k=0, \ldots, d$, there is a polynomial $p_{k}$ of degree $k$ with its leading coefficient 
equal to $1 / k$ ! such that for all $\alpha \in \mathbb{Z}$

$$
\sum_{\beta \in \mathbb{Z}} A(\alpha-2 \beta) v_{k}(\beta)=v_{k}(\alpha) / 2^{k}
$$

where $v_{k}(\alpha)=\left(p_{k}(\alpha), p_{k}^{\prime}(\alpha), \ldots, p_{k}^{(d)}(\alpha)\right)^{T}$.

Definition $5 A C^{d}$ function $\phi$ is reproduced by a $C^{d}$ Hermite subdivision scheme if it is the limit function associated with a sequence of refinements $f_{n}$ of this scheme.

Theorem 8 We consider a Hermite subdivision scheme of degree d which satisfies the spectral condition, then any polynomial of degree less than or equal to $d$ is reproduced by the scheme.

Proof: For $k \in\{0, \ldots, d\}$, let $p_{k}(x)$ be the polynomial defined in Theorem 7 which provides the eigenvector $v_{k}$ appearing in (12). We set $f_{0}(\alpha)=\left(p_{k}(\alpha), p_{k}^{\prime}(\alpha), \ldots, p_{k}^{(d)}(\alpha)\right)^{T}$, then by (1) and (12), we get by induction that $D^{n} f_{n}=f_{0} / 2^{k n}$ and $f_{n}^{(i)}(\alpha) / 2^{i n}=$ $p_{k}^{(i)}(\alpha) / 2^{k n}, i=0,1, \ldots, d$, for $n=1,2,3, \ldots$. Let $\sum_{\ell=0}^{k} c_{\ell} x^{\ell} / \ell$ ! be the Taylor expansion of $p_{k}$, we will show that the limit function associated with the refinements $f_{n}$ is $\phi(x)=c_{k} x^{k} / k$ !. We distinguish 3 cases.

Case $i>k: f_{n}^{(i)}(\alpha)=p_{k}^{(i)}(\alpha) 2^{(i-k) n}=0, \phi^{(i)}\left(\alpha / 2^{n}\right)=0$ so that $f_{n}^{(i)}(\alpha)-\phi^{(i)}\left(\alpha / 2^{n}\right)=$ 0 ,

Case $i=k: f_{n}^{(k)}(\alpha)=p_{k}^{(k)}(\alpha)=c_{k}, \phi^{(k)}\left(\alpha / 2^{n}\right)=c_{k}$ and again, $f_{n}^{(k)}(\alpha)-\phi^{(k)}\left(\alpha / 2^{n}\right)=$ 0 ,

Case $i<k$ : then $f_{n}^{(i)}(\alpha)=2^{(i-k) n} p_{k}^{(i)}(\alpha)=2^{(i-k) n} \sum_{\ell=i}^{k} c_{\ell} \alpha^{\ell-i} /(\ell-i)$ !, $\phi^{(i)}\left(\alpha / 2^{n}\right)=$ $2^{(i-k) n} c_{k} \alpha^{k-i} /(k-i)$ ! and $f_{n}^{(i)}(\alpha)-\phi^{(i)}\left(\alpha / 2^{n}\right)=2^{(i-k) n} \sum_{\ell=i}^{k-1} c_{\ell} \alpha^{\ell-i} /(\ell-i)$ !. Finally $f_{n}^{(i)}(\alpha)-\phi^{(i)}\left(\alpha / 2^{n}\right)=O\left(1 / 2^{n}\right)$ uniformly for $\alpha \in\left[-L 2^{n}, L 2^{n}\right]$ as $n \rightarrow \infty$.

According to Definition 1, $\phi$ is the limit function associated with the refinements $f_{n}$. The polynomial $x^{k}$ is reproduced and by linearity, any polynomial of degree less or equal to $d$ is reproduced by the scheme.

Corollary 9 A Hermite subdivision scheme of degree $d$ which satisfies the spectral condition is nondegenerate.

The function $x^{d}$ is the associated limit function of refinements $f_{n}$. In Definition 3 , take these refinements and $a=1$. 


\section{Associated subdivision scheme}

Given a Hermite subdivision scheme $\mathcal{H}$ satisfying the spectral condition, we will associate a vector subdivision scheme $\mathcal{S}$, the so-called Taylor subdivision scheme. We begin by recalling what a vector subdivision scheme is and when it is affine.

Definition $6 A$ vector subdivision matrix of order $p$ is a matrix function $S: \mathbb{Z}^{2} \rightarrow$ $\mathbb{R}^{p \times p}$ for which there exists an interval $\left[\sigma, \sigma^{\prime}\right], \sigma, \sigma^{\prime} \in \mathbb{Z}, \sigma \leq \sigma^{\prime}$ such that $S(\alpha, \beta)=0$ whenever $\alpha-2 \beta \notin\left[\sigma, \sigma^{\prime}\right]$. If $g_{0}: \mathbb{R}^{p} \rightarrow \mathbb{R}$, then the recursive formula

$$
g_{n+1}(\alpha)=\sum_{\beta \in \mathbb{Z}} S(\alpha, \beta) g_{n}(\beta)
$$

generates the refinements of $g_{0}$ and defines a vector subdivision scheme $\mathcal{S}$ of order $p$. The interval $\left[\sigma, \sigma^{\prime}\right]$ is a support of $\mathcal{S}$. If there exists a matrix function $B: \mathbb{Z} \rightarrow \mathbb{R}^{p \times p}$ such that $S(\alpha, \beta)=B(\alpha-2 \beta)$, then $B$ is the mask of $\mathcal{S}$.

Definition 7 A vector subdivision matrix $S$ (and its corresponding vector subdivision scheme) is affine if $\sum_{\beta \in \mathbb{Z}} \sum_{j=1}^{p} s_{i j}(\alpha, \beta)=1, \alpha \in \mathbb{Z}, i=1,2, \ldots, p$, where $s_{i j}(\alpha, \beta)$ are the entries of $S(\alpha, \beta)$. The subdivision scheme is scalar if $p=1$.

For $f=\left(f^{(0)}, \ldots, f^{(d)}\right)^{T}$ where $f^{(i)}: \mathbb{Z} \rightarrow \mathbb{C}, i=0, \ldots, d$, we define the operator $P f=g=\left(g^{(0)}, \ldots, g^{(d)}\right)^{T}$ where

$$
\left.\begin{array}{l}
g^{(0)}(\alpha)=f^{(d)}(\alpha) \\
\left.g^{(i)}(\alpha)=i !\left(f^{(d-i)}(\alpha+1)-\sum_{j=1}^{i} \frac{f^{(d-j)}(\alpha)}{(i-j) !}\right), \quad i=1, \ldots, d\right)
\end{array}\right\}
$$

If $P_{0}, P_{1}$ are the respective square matrices of order $d+1$

$$
\left(\begin{array}{cccccc}
0 & 0 & 0 & \cdots & 0 & 1 \\
0 & 0 & 0 & \cdots & -1 & 0 \\
\vdots & \vdots & \vdots & \cdots & \vdots & \vdots \\
0 & 0 & -\frac{(d-2) !}{0 !} & \cdots & -\frac{(d-2) !}{(d-3) !} & 0 \\
0 & -\frac{(d-1) !}{0 !} & -\frac{(d-1) !}{1 !} & \cdots & -\frac{(d-1) !}{(d-2) !} & 0 \\
-\frac{d !}{0 !} & -\frac{d !}{1 !} & -\frac{d !}{2) !} & \cdots & -\frac{d !}{(d-1) !} & 0
\end{array}\right),\left(\begin{array}{cccccc}
0 & 0 & 0 & \cdots & 0 & 0 \\
0 & 0 & 0 & \cdots & 1 ! & 0 \\
\vdots & \vdots & \vdots & \cdots & \vdots & \\
0 & 0 & (d-2) ! & \cdots & 0 & 0 \\
0 & (d-1) ! & 0 & \cdots & 0 & 0 \\
d ! & 0 & 0 & \cdots & 0 & 0
\end{array}\right),
$$

then

$$
P f(\alpha)=P_{0} f(\alpha)+P_{1} f(\alpha+1)
$$


Theorem 10 Let $\mathcal{H}$ be a Hermite subdivision scheme of degree d which satisfies the spectral condition and whose mask $A(\alpha)=\left(a_{i j}(\alpha)\right)_{i, j=0, \ldots, d}$ has support included in $\left[\sigma, \sigma^{\prime}\right]$. Let $f_{n}=\left(f_{n}^{(0)}, f_{n}^{(1)}, \ldots, f_{n}^{(d)}\right)^{T}, n=0,1,2, \ldots$ be the refinements of a $\mathcal{H}$, we set $g_{n}=2^{\text {nd }} P D^{n} f_{n}$ and $C(\alpha)=2^{d}\left(P_{0} A(\alpha)+P_{1} A(\alpha+1)\right)$ where $P, P_{0}, P_{1}$ are defined in (13-14). If we define the matrix function $B(\alpha)=\left(b_{i j}(\alpha)\right)_{i, j=0, \ldots, d}$ by

$$
\begin{array}{r}
b_{i, d-j}(\alpha)=\frac{1}{(d-j) !} \sum_{\beta=1}^{\infty} \sum_{k=0}^{j} c_{i, k}(\alpha-2 \beta) \frac{(\beta-1)^{j-k}}{(j-k) !}, j=0, \ldots, d-1 \\
b_{i, 0}(\alpha)=c_{i d}(\alpha)
\end{array}
$$

for $i=0, \ldots, d$, then the support of $B$ is contained in $\left[\sigma-1, \sigma^{\prime}\right]$ and the sequence $g_{n}$ is the sequence of refinements of an affine vector subdivision scheme $\mathcal{S}$ whose mask is $B$.

Proof: Let us find a condition that a matrix function $B$ should satisfy in order that the functions $g_{n}=2^{n d} P D^{n} f_{n}$ are the refinements of a vector subdivision scheme of mask $B$.

$$
\begin{array}{r}
g_{n+1}(\alpha)=\sum_{\beta \in \mathbb{Z}} B(\alpha-2 \beta) g_{n}(\beta)=2^{n d} \sum_{\beta \in \mathbb{Z}} B(\alpha-2 \beta) P D^{n} f_{n}(\beta) \\
g_{n+1}(\alpha)=2^{(n+1) d} P D^{n+1} f_{n+1}(\alpha)=2^{(n+1) d} \sum_{\beta \in \mathbb{Z}} P A(\alpha-2 \beta) D^{n} f_{n}(\beta) .
\end{array}
$$

Since

$$
\begin{array}{r}
\sum_{\beta \in \mathbb{Z}} B(\alpha-2 \beta) P D^{n} f_{n}(\beta)=\sum_{\beta \in \mathbb{Z}} B(\alpha-2 \beta)\left[P_{0} D^{n} f_{n}(\beta)+P_{1} D^{n} f_{n}(\beta+1)\right] \\
=\sum_{\beta \in \mathbb{Z}}\left[B(\alpha-2 \beta) P_{0}+B(\alpha+2-2 \beta) P_{1}\right] D^{n} f_{n}(\beta),
\end{array}
$$

such a condition is

$$
B(\alpha) P_{0}+B(\alpha+2) P_{1}=C(\alpha)=2^{d}\left(P_{0} A(\alpha)+P_{1} A(\alpha+1)\right) .
$$

Let us remark that the support of $C$ is included in $\left[\sigma-1, \sigma^{\prime}\right]$.

When comparing the $j$ th column of $B(\alpha) P_{0}+B(\alpha+2) P_{1}$ with that of $C$ and using the definition of $P_{0}$ and $P_{1}$, we get the following equations:

$$
\begin{array}{r}
b_{i, d-j}(\alpha+2)=\frac{c_{i, j}(\alpha)}{(d-j) !}+\sum_{k=0}^{j}\left(\begin{array}{l}
d-k \\
d-j
\end{array}\right) b_{i, d-k}(\alpha), \quad j=0,1, \ldots, d-1 \\
b_{i, 0}(\alpha)=c_{i, d}(\alpha) .
\end{array}
$$


Both Equations (16) and (18) are the same. In order to solve (17), we note that $C(\alpha)=0$ if $\alpha<\sigma-1$ and we set $B(\alpha)=0$ for $\alpha<\sigma-1$. Obviously, the functions $b_{i, d-j}(\alpha+2), i=0,1, \ldots, d, j=0,1, \ldots, d-1$ can be computed recursively for $\alpha=\sigma-1, \sigma, \sigma+1, \ldots$. Let us check that (15) is a solution of (17).

We define the matrix function $B$ by (15-16). Matrix $B$ is well defined and has the property that $B(\alpha)=0$ for $\alpha<\sigma-1$ since $C(\alpha)=0$ if $\alpha<\sigma-1$. Let $i \in\{0,1 \ldots, d\}$, $j \in\{0,1 \ldots, d-1\}$ and $\alpha \in \mathbb{Z}$, then, as the support of $C$ is bounded,

$$
\begin{array}{r}
(d-j) ! \sum_{k=0}^{j}\left(\begin{array}{l}
d-k \\
d-j
\end{array}\right) b_{i, d-k}(\alpha)=\sum_{k=0}^{j} \sum_{\beta=1}^{\infty} \sum_{\ell=0}^{k} \frac{c_{i, \ell}(\alpha-2 \beta)(\beta-1)^{k-\ell}}{(j-k) !(k-\ell) !} \\
=\sum_{\beta=1}^{\infty} \sum_{\ell=0}^{j} \sum_{k=0}^{j-\ell} \frac{c_{i, \ell}(\alpha-2 \beta)(\beta-1)^{k}}{(j-\ell-k) ! k !}=\sum_{\beta=1}^{\infty} \sum_{\ell=0}^{j} \frac{c_{i, \ell}(\alpha-2 \beta) \beta^{j-\ell}}{(j-\ell) !}
\end{array}
$$

since $\sum_{k=0}^{j-\ell} \frac{(j-\ell) !}{(j-\ell-k) ! k !}(\beta-1)^{k}=(\beta-1+1)^{j-\ell}=\beta^{j-\ell}$ (from binomial theorem).

Consequently, we get

$$
\begin{array}{r}
c_{i, j}(\alpha)+(d-j) ! \sum_{k=0}^{j}\left(\begin{array}{l}
d-k \\
d-j
\end{array}\right) b_{i, d-k}(\alpha)=\sum_{\beta=0}^{\infty} \sum_{\ell=0}^{j} \frac{c_{i, \ell}(\alpha-2 \beta) \beta^{j-\ell}}{(j-\ell) !} \\
=\sum_{\beta=1}^{\infty} \sum_{\ell=0}^{j} \frac{c_{i, \ell}(\alpha+2-2 \beta)(\beta-1)^{j-\ell}}{(j-\ell) !}=(d-j) ! b_{i, d-j}(\alpha+2) .
\end{array}
$$

(17) is satisfied and the sequence $g_{n}$ is the sequence of refinements of the vector subdivision scheme whose mask is $B$.

Before studying the support of $B$, let us show that

$$
\sum_{\beta \in \mathbb{Z}} \sum_{k=0}^{j} \frac{(\beta-1)^{j-k}}{(j-k) !} c_{i, k}(\alpha-2 \beta)=0, j=0, \ldots, d-1
$$

We use the basis of $\mathcal{P}_{d-1}$ composed with the polynomials $p_{k}$ of degree $k, k=$ $0, \ldots, d-1$ such that the vector function $v_{k}(\alpha)=\left(p_{k}(\alpha), \ldots, p_{k}^{(d)}(\alpha)\right)^{T}$ is an eigenvector of the supermatrix $H=(A(\alpha-2 \beta))_{\alpha, \beta \in \mathbb{Z}}$ for the eigenvalue $1 / 2^{k}$. We have

$$
P_{0} \sum_{\beta \in \mathbb{Z}} A(\alpha-2 \beta) v_{k}(\beta)+P_{1} \sum_{\beta \in \mathbb{Z}} A(\alpha+1-2 \beta) v_{k}(\beta)=\frac{1}{2^{k}}\left(P_{0} v_{k}(\alpha)+P_{1} v_{k}(\alpha+1)\right) \text {. }
$$

From Lemma 3 , we obtain $P_{0} v_{k}(\alpha)+P_{1} v_{k}(\alpha+1)=0$ and $\sum_{\beta \in \mathbb{Z}} C(\alpha-2 \beta) v_{k}(\beta)=0$. 
For $j \in\{0,1, \ldots, d-1\}$, the vector function $w_{j}(\alpha)=\left(q(\alpha), q^{\prime}(\alpha), \ldots, q^{(d)}(\alpha)\right)^{T}$ where $q(\alpha)=(\alpha-1)^{j}$ is a linear combination of $\left\{v_{k}\right\}_{k=0,1, \ldots, d-1}$ and $\sum_{\beta \in \mathbb{Z}} C(\alpha-2 \beta) w_{j}(\beta)=$ 0 , i.e. (19) is satisfied.

Let us study the support of $B$. Since the support of $C$ is included in $\left[\sigma-1, \sigma^{\prime}\right]$, from (15)-(16), we deduce that the support of $B$ is included in $[\sigma-1,+\infty)$. Let $\alpha>\sigma^{\prime}$, then $c_{i j}(\alpha-2 \beta)=0$ for $i, j=0,1, \ldots, d$ and for $\beta<1$. ¿From that and from (15)-(19) we obtain $b_{i, d-j}(\alpha)=0$ for $j=0, \ldots, d-1$ and for $i=0, \ldots, d$. Moreover, from (16), we obviously get $b_{i, 0}(\alpha)=0$ for $i=0, \ldots, d$. The support of $B$ is contained in $\left[\sigma-1, \sigma^{\prime}\right]$.

To prove that the vector subdivision scheme $\mathcal{S}$ whose mask is $B$ is affine, we use the eigenvalue $1 / 2^{d}$ of $H$ and the associated eigenvector built from the vector function $v_{d}(\alpha)=\left(p_{d}(\alpha), \ldots, p_{d}^{(d)}(\alpha)\right)^{T}$ where $p_{d}$ is a polynomial of degree $d$ which can be chosen such that its leading coefficient is $1 / d$ !.

If we define $f_{0}=v_{d}$, then for all $\alpha \in \mathbb{Z}$,

$$
D f_{1}(\alpha)=\sum_{\beta \in \mathbb{Z}} A(\alpha-2 \beta) f_{0}(\beta)=f_{0}(\alpha) / 2^{d} .
$$

We get $g_{1}(\alpha)=2^{d} P D f_{1}(\alpha)=P f_{0}(\alpha)=g_{0}(\alpha)$. From Lemma 3, we deduce $g_{0}(\alpha)=$ $P v_{d}(\alpha)=(1, \ldots, 1)^{T}$. Since $g_{1}(\alpha)=\sum_{\beta \in \mathbb{Z}} B(\alpha-2 \beta) g_{0}(\alpha)=g_{0}(\alpha)$, we obtain the last result of the Theorem.

Definition 8 The vector subdivision scheme with mask $B$ is called the Taylor subdivision scheme associated with $\mathcal{H}$.

\section{Criterion of convergence for a Hermite scheme}

We will propose a sufficient condition for $C^{d}$ convergence of a Hermite subdivision scheme of degree $d$. This criterion is that the subdivision scheme of the finite differences arising from the Taylor subdivision scheme is contractive. Before reaching the main theorem, we need a lemma.

Lemma 11 Let $f_{n}: \mathbb{Z} \rightarrow \mathbb{R}$ be a sequence of functions. We assume that $\lim _{n \rightarrow \infty} f_{n}(0)=$ $c$ and that there exists a continuous function $\psi: \mathbb{R} \rightarrow \mathbb{R}$ such that for every $L>0$, $2^{n} \Delta f_{n}(\alpha)-\psi\left(\alpha / 2^{n}\right)=o(1)$ uniformly for $\alpha \in\left[-L 2^{n}, L 2^{n}\right]$ as $n \rightarrow \infty$. Then for every $L>0, f_{n}(\alpha)-c-\int_{0}^{\alpha / 2^{n}} \psi(t) d t=o(1)$ uniformly for $\alpha \in\left[-L 2^{n}, L 2^{n}\right]$ as $n \rightarrow \infty$.

Proof: Let $\varepsilon>0$ and let $L>0$, there exists an integer $N_{1}>0$ such that

$$
\left(\forall n>N_{1}\right)\left(\forall \beta \in \mathbb{Z} \cap\left[-L 2^{n}, L 2^{n}\right]\right) \quad\left|2^{n} \Delta f_{n}(\beta)-\psi\left(\beta / 2^{n}\right)\right|<\varepsilon .
$$


Now since $\psi$ is uniformly continuous on $[-L, L]$, there exists $N_{2} \geq N_{1}$ such that $\left(\forall n>N_{2}\right)\left(\forall \beta \in \mathbb{Z} \cap\left[-L 2^{n}, L 2^{n}-1\right]\right)$,

$$
\left|\psi\left(\beta / 2^{n}\right) / 2^{n}-\int_{\beta / 2^{n}}^{(\beta+1) / 2^{n}} \psi(t) d t\right|<\varepsilon / 2^{n}
$$

For $n>N_{2}$, let $\alpha \in\left[0, L 2^{n}\right] \cap \mathbb{Z}$. As $f_{n}(\alpha)=f_{n}(0)+\sum_{\beta=0}^{\alpha-1} \Delta f_{n}(\beta)$, with (20), we obtain

$$
\left|f_{n}(\alpha)-c-\sum_{\beta=0}^{\alpha-1} \psi\left(\beta / 2^{n}\right) / 2^{n}\right| \leq\left|f_{n}(0)-c\right|+L \varepsilon .
$$

From (21), we get

$$
\left|\sum_{\beta=0}^{\alpha-1} \psi\left(\beta / 2^{n}\right) / 2^{n}-\int_{0}^{\alpha / 2^{n}} \psi(t) d t\right| \leq \sum_{\beta=0}^{\alpha-1}\left|\psi\left(\beta / 2^{n}\right) / 2^{n}-\int_{\beta / 2^{n}}^{(\beta+1) / 2^{n}} \psi(t) d t\right|<L \varepsilon .
$$

It follows from both previous inequalities that

$$
\left|f_{n}(\alpha)-c-\int_{0}^{\alpha / 2^{n}} \psi(t) d t\right| \leq\left|f_{n}(0)-c\right|+2 L \varepsilon
$$

We have a similar result for $\alpha \in\left[-L 2^{n}, 0\right] \cap \mathbb{Z}$. Let $N>N_{2}$ be such that $\left|f_{n}(0)-c\right|<\varepsilon$ whenever $n>N$. We have proved $(\forall n>N)\left(\forall \alpha \in\left[-L 2^{n}, L 2^{n}\right] \cap \mathbb{Z}\right)$

$$
\left|f_{n}(\alpha)-c-\int_{0}^{\alpha / 2^{n}} \psi(t) d t\right| \leq(2 L+1) \varepsilon
$$

and the conclusion can easily be deduced.

Definition 9 We say that a vector subdivision scheme $\mathcal{S}$ of order $p$ is $C^{0}$ if for every sequence of refinements $f_{n}: \mathbb{Z} \rightarrow \mathbb{R}^{p}$ of $\mathcal{S}$, there is a continuous function $\phi: \mathbb{R} \rightarrow \mathbb{R}^{p}$ such that for every $\epsilon>0$ and for every $L>0$, there exists $N$ such that $\left\|f_{n}(\alpha)-\phi\left(\alpha / 2^{n}\right)\right\|<\epsilon$ for every $n>N$ and every $\alpha \in\left[-L 2^{n}, L 2^{n}\right]$. The function $\phi$ is called the limit function associated with the refinements $f_{n}$. If for any limit function, all its components are the same, we say that $\mathcal{S}$ is $C^{0}$ with equal components.

Theorem 12 Let $\mathcal{H}$ be a Hermite subdivision scheme of order d which satisfies the spectral condition. Let $B(\alpha)=\left(b_{i j}(\alpha)\right)_{i, j=0,1, \ldots, d}$ be the mask of the Taylor subdivision scheme $\mathcal{S}$ associated with $\mathcal{H}$. We define the functions $s, t: \mathbb{Z}^{2} \rightarrow \mathbb{R}$

$$
s((d+1) \alpha+i,(d+1) \beta+j)=b_{i j}(\alpha-2 \beta), \quad \alpha, \beta \in \mathbb{Z}, i, j=0,1, \ldots, d,
$$




$$
t(\alpha, \beta)=-\sum_{\gamma=-\infty}^{\beta}[s(\alpha+1, \gamma)-s(\alpha, \gamma)]=\sum_{\gamma=\beta+1}^{\infty}[s(\alpha+1, \gamma)-s(\alpha, \gamma)]
$$

If, moreover, there is an integer $n$ such that

$$
\max \left\{\sum_{\beta \in \mathbb{Z}}\left|t_{n}(\alpha, \beta)\right|: \alpha \in\left[0,(d+1) 2^{n}-1\right]\right\}<1
$$

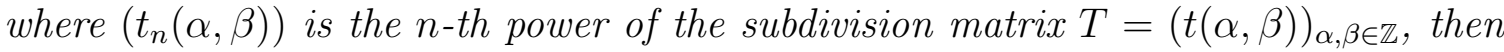
the Taylor subdivision scheme $\mathcal{S}$ is $C^{0}$ with equal components and the Hermite subdivision scheme $\mathcal{H}$ is $C^{d}$.

Proof: In the first part, we show that the Taylor subdivision scheme $\mathcal{S}$ is $C^{0}$. Let $g_{n}=\left(g_{n}^{(0)}, g_{n}^{(1)}, \ldots, g_{n}^{(d)}\right)^{T}, n=0,1,2, \ldots$ be the refinements of $\mathcal{S}$, we define the sequence $h_{n}: \mathbb{Z} \rightarrow \mathbb{R}$ by setting $h_{n}((d+1) \alpha+i)=g_{n}^{(i)}(\alpha), \alpha \in \mathbb{Z}, i=0,1, \ldots, d$. There is no essential difference between $g_{n}$ and $h_{n}$. $h_{n}$ is the sequence of refinements of a non-uniform binary subdivision scheme whose subdivision matrix $S=(s(\alpha, \beta))_{\alpha, \beta \in \mathbb{Z}}$ is given by $(22)$. The refinement rule is $h_{n+1}(\alpha)=\sum_{\beta} s(\alpha, \beta) h_{n}(\beta)$. We still call this scheme $\mathcal{S}$. From Theorem 10, the subdivision matrix is affine: for every $\alpha \in \mathbb{Z}$, $\sum_{\beta} s(\alpha, \beta)=1$.

Since $S$ is affine, we can define the subdivision matrix $T=(t(\alpha, \beta))$ with $(23)$ (see Equations (3.7) and (3.8) of [1] or Proposition 10 of [3]). As it is shown in [3], the sequence of the finite differences $\Delta h_{n}=h_{n}(\alpha+1)-h_{n}(\alpha)$ is the sequence of refinements of the subdivision scheme whose subdivision matrix is $T$.

The subdivision matrix $T$ (like $S$ ) is periodic of period $d+1$, i.e. $t(\alpha+2 d+2, \beta+$ $d+1)=t(\alpha, \beta)$. Let $t_{n}(\alpha, \beta)$ the entries of the matrix $T^{n}$, then $T^{n}$ is periodic with the following meaning

$$
t_{n}\left(\alpha+(d+1) 2^{n}, \beta+d+1\right)=t_{n}(\alpha, \beta) .
$$

We verify this periodicity recursively through the following equations:

$$
\begin{aligned}
\left.t_{n+1}\left(\alpha+(d+1) 2^{n+1}, \beta+d+1\right)\right) & =\sum_{\gamma \in \mathbb{Z}} t\left(\alpha+(d+1) 2^{n+1}, \gamma\right) t_{n}(\gamma, \beta+d+1) \\
& =\sum_{\gamma \in \mathbb{Z}} t\left(\alpha, \gamma-(d+1) 2^{n}\right) t_{n}(\gamma, \beta+d+1) \\
& =\sum_{\gamma \in \mathbb{Z}} t(\alpha, \gamma) t_{n}\left(\gamma+(d+1) 2^{n}, \beta+d+1\right) \\
& =\sum_{\gamma \in \mathbb{Z}} t(\alpha, \gamma) t_{n}(\gamma, \beta)=t_{n+1}(\alpha, \beta)
\end{aligned}
$$


From (24) and from (25), we obtain that the matrix $T^{n}$ is contractive:

$$
\left.\left\|T^{n}\right\|_{\infty}=\sup \sum_{\beta \in \mathbb{Z}}\left|t_{n}(\alpha, \beta)\right|: \alpha \in \mathbb{Z}\right\}<1 .
$$

This inequality is a criterion for the $C^{0}$ convergence of $\mathcal{S}$ (see Theorem 3.3 of [1], Theorem 2.4 of [11], Theorem 3.2 of [12] or Theorem 4 of [6]). The subdivision scheme $\mathcal{S}$ is $C^{0}$.

Let $\theta$ be the limit function of the refinements $h_{n}$ and let $L>0$, then $h_{n}(\alpha)-$ $\theta\left(\alpha / 2^{n}\right)=o(1)$ uniformly for $\alpha \in\left[-L 2^{n}, L 2^{n}\right]$. We set $\psi(x)=\theta((d+1) x)$ and we get that for $i=0,1, \ldots, d, g_{n}^{(i)}(\alpha)-\psi\left(\alpha / 2^{n}\right)$ is the sum of the two terms $h_{n}((d+$ 1) $\alpha+i)-\theta\left(((d+1) \alpha+i) / 2^{n}\right)$ and $\theta\left(((d+1) \alpha+i) / 2^{n}\right)-\theta\left((d+1) \alpha / 2^{n}\right)$. Let $L>0$, then $g_{n}(\alpha)-\psi\left(\alpha / 2^{n}\right)=o(1)$ uniformly for $\alpha \in\left[-L 2^{n}, L 2^{n}\right]$. The limit of the sequence of refinement $g_{n}$ is the vector function $(\psi(x), \psi(x), \ldots, \psi(x))^{T}$ with $d+1$ equal components. The Taylor subdivision scheme $\mathcal{S}$ associated with $\mathcal{H}$ is $C^{0}$ with equal components.

In this second part of the proof, we prove that the Hermite subdivision scheme $\mathcal{H}$ is $C^{d}$. Let $f_{n}=\left(f_{n}^{(0)}, f_{n}^{(1)}, \ldots, f_{n}^{(d)}\right)^{T}$ be a sequence of refinements of $\mathcal{H}$ and let $g_{n}=\left(g_{n}^{(0)}, g_{n}^{(1)}, \ldots, g_{n}^{(d)}\right)^{T}=2^{d n} P D^{n} f_{n}$ be the corresponding sequence of refinements of $\mathcal{S}$, we have

$$
\left.\begin{array}{l}
g_{n}^{(d-i)}(\alpha)=2^{d n}(d-i) !\left(f_{n}^{(i)}(\alpha+1) / 2^{i n}-\sum_{j=i}^{d-1} \frac{f_{n}^{(j)}(\alpha) / 2^{j n}}{(j-i) !}\right), \quad i=0, \ldots, d-1 \\
g_{n}^{(0)}(\alpha)=f_{n}^{(d)}(\alpha) .
\end{array}\right\} .
$$

Since $\mathcal{S}$ is $C^{0}$ with equal components, there exists a continuous function $\psi: \mathbb{R} \rightarrow \mathbb{R}$ such that for every $L>0, g_{n}^{(i)}(\alpha)-\psi\left(\alpha / 2^{n}\right)=o(1), i=0,1, \ldots, d$ uniformly for every $\alpha \in\left[-L 2^{n}, L 2^{n}\right]$ as $n \rightarrow \infty$.

Then

$$
f_{n}^{(d)}(\alpha)-\psi\left(\alpha / 2^{n}\right)=o(1)
$$

and for $i=0, \ldots, d-1$,

$$
f_{n}^{(i)}(\alpha+1) / 2^{i n}-\sum_{j=i}^{d-1} \frac{1}{(j-i) !} f_{n}^{(j)}(\alpha) / 2^{j n}-\psi\left(\alpha / 2^{n}\right) /(d-i) !=o\left(1 / 2^{d n}\right)
$$

uniformly for every $\alpha \in\left[-L 2^{n}, L 2^{n}\right]$ as $n \rightarrow \infty$.

By Theorem 7 , for $i=0,1, \ldots d$, the sequence $f_{n}^{(i)}(0)$ converges as $n \rightarrow \infty$, and we set $c_{i}=\lim _{n \rightarrow \infty} f_{n}^{(i)}(0)$. Then we define

$$
\phi(x)=\sum_{k=0}^{d-1} c_{k} x^{k}+\frac{1}{(d-1) !} \int_{0}^{x}(x-t)^{d-1} \psi(t) d t .
$$


Let $k \in\{0,1, \ldots, d\}$, we consider Property $\left(\mathrm{P}_{k}\right)$ : for every $\epsilon>0$ and for every $L>0$, there exists $N$ such that $\left|f_{n}^{(k)}(\alpha)-\phi^{(k)}\left(\alpha / 2^{n}\right)\right|<\epsilon$ for every $n>N$ and for every $\alpha \in\left[-L 2^{n}, L 2^{n}\right]$. We proceed with a backward finite recursion, first showing $\left(\mathrm{P}_{d}\right)$, then $\left(\mathrm{P}_{d-1}\right), \ldots$, down to $\left(\mathrm{P}_{0}\right)$.

$\left(\mathrm{P}_{d}\right)$ is $(26)$ since $\psi(x)=\phi^{(d)}(x)$.

For Property $\left(\mathrm{P}_{d-1}\right)$, we use $(27)$ with $i=d-1$ and we obtain that the sequence $2^{n} \Delta f_{n}^{(d-1)}(\alpha)-\psi\left(\alpha / 2^{n}\right)=o(1)$. Since the sequence $f_{n}^{(d-1)}(0)$ converges, using Lemma 11 , we conclude that the sequence

$$
f_{n}^{(d-1)}(\alpha)-c_{d-1}-\int_{0}^{\alpha / 2^{n}} \psi(t) d t=f_{n}^{(d-1)}(\alpha)-\phi^{(d-1)}\left(\alpha / 2^{n}\right)=o(1)
$$

uniformly for every $\alpha \in\left[-L 2^{n}, L 2^{n}\right]$ as $n \rightarrow \infty$.

Now let us assume $\left(\mathrm{P}_{d}\right), \ldots,\left(\mathrm{P}_{k+1}\right)$ for $k \in\{0, \ldots, d-1\}$. Again, we use $(27)$ with $i=k$ and we obtain that the sequence

$$
\Delta f_{n}^{(k)}(\alpha) / 2^{k n}-\sum_{j=k+1}^{d-1} \frac{f_{n}^{(j)}(\alpha) / 2^{n j}}{(j-k) !}-\frac{\psi\left(\alpha / 2^{n}\right) / 2^{d n}}{(d-k) !}=o\left(1 / 2^{d n}\right)
$$

uniformly for every $\alpha \in\left[-L 2^{n}, L 2^{n}\right]$. We multiply the previous sequence by $2^{(k+1) n}$ and we know from the recursion hypotheses that the sequences $f_{n}^{(j)}(\alpha)$ are uniformly bounded for $j>k+1$ and $f_{n}^{(k+1)}(\alpha)-\phi_{k+1}\left(\alpha / 2^{n}\right)=o(1)$ uniformly for every $\alpha \in$ $\left[-L 2^{n}, L 2^{n}\right]$ as $n \rightarrow \infty$. We deduce that the sequence $2^{n} \Delta f_{n}^{(k)}(\alpha)-\phi_{k}\left(\alpha / 2^{n}\right)$ is $o(1)$. Since the sequence $f_{n}^{(d-1)}(0)$ converges, using Lemma 11 again, we conclude that $f_{n}^{(k)}(\alpha)-\phi^{(k)}\left(\alpha / 2^{n}\right)=o(1)$ uniformly for every $\alpha \in\left[-L 2^{n}, L 2^{n}\right]$ as $n \rightarrow \infty$, which is Property $\left(\mathrm{P}_{k}\right)$.

Then $\left(\mathrm{P}_{k}\right)$ is true for $k=0, \ldots, d$, i.e. $\mathcal{H}$ is $C^{d}$.

\section{Examples of Hermite subdivision schemes}

For every positive integer $d$, we consider two specific Hermite subdivision schemes, the first one is interpolatory and is obtained by solving a Hermite problem, the second one is a kind of corner cutting. For each of these schemes, we consider the corresponding Taylor subdivision scheme $\mathcal{S}$ and the subdivision scheme of finite differences $\mathcal{T}$ with their subdivision matrices $S, T$.

Before the definitions, we recall the Obreshkov interpolation polynomial [15]. Let $d, e \geq 0$ and let $y=\left(y^{(0)}, y^{(1)}, \ldots, y^{(d)}\right)$ and $z=\left(z^{(0)}, z^{(1)}, \ldots, z^{(e)}\right)$ be two vectors, one from $\mathbb{R}^{d+1}$, the other from $\mathbb{R}^{e+1}$, the polynomial $p$ of degree $2 d+1$ that satisfies

$$
p^{(i)}(a)=y^{(i)}, i=0,1, \ldots, d ; \quad p^{(i)}(b)=z^{(i)}, i=0,1, \ldots, e
$$


is the Obreshkov interpolation polynomial

$$
\begin{aligned}
p(x) & =\sum_{j=0}^{d} y^{(j)} \frac{(x-b)^{e+1}}{j !(a-b)^{e+1}} \sum_{k=0}^{d-j}\left(\begin{array}{c}
e+k \\
k
\end{array}\right) \frac{(x-a)^{j+k}}{(b-a)^{k}} \\
& +\sum_{j=0}^{e} z^{(j)} \frac{(x-a)^{d+1}}{j !(b-a)^{d+1}} \sum_{k=0}^{e-j}\left(\begin{array}{c}
d+k \\
k
\end{array}\right) \frac{(x-b)^{j+k}}{(a-b)^{k}} .
\end{aligned}
$$

This formula is also given in the contribution of Sendov and Andrew [16], p.403. We limit ourselves to the case $e=d$. We define

$$
u_{j}(t)=\frac{1}{j !} \sum_{k=0}^{d-j}\left(\begin{array}{c}
d+k \\
k
\end{array}\right) t^{j+k}(1-t)^{d+1}, \quad v_{j}(t)=(-1)^{j} u_{j}(1-t), \quad j=0,1, \ldots, d .
$$

By substitution and differentiation, with $t=(x-a) /(b-a)$, we obtain

$$
p^{(i)}(a+(b-a) t)(b-a)^{i}=\sum_{j=0}^{d}\left[y^{(j)} u_{j}^{(i)}(t)+z^{(j)} v_{j}^{(i)}(t)\right](b-a)^{j}, \quad i=0,1, \ldots d .
$$

If we define the two matrices $U(t)=\left(u_{j}^{(i)}(t)\right)_{i, j=0,1, \ldots, d}$ and $V(t)=\left(v_{j}^{(i)}(t)\right)_{i, j=0,1, \ldots, d}$, the matrix form of the previous equation is

$$
D(b-a) f(a+(b-a) t)=U(t) D(b-a) y+V(t) D(b-a) z
$$

where $f=\left(p, p^{\prime}, \ldots, p^{(d)}\right)^{T}$ and $D(h)$ is the diagonal matrix whose diagonal is $1, h, \ldots, h^{d}$, (here $h=b-a)$. We also remark that

$$
V(t)=S U(1-t) S
$$

where $S$ is the diagonal matrix whose diagonal is $1,-1, \ldots,(-1)^{d}$.

\subsection{Hermite interpolation scheme: HIS}

Let $d>0$ and let $w: \mathbb{Z} \rightarrow \mathbb{R}^{d+1}$ be a family of Hermite data. Let us consider the unique piecewise polynomial $\phi: \mathbb{R} \rightarrow \mathbb{R}$ such that on every interval $[\alpha, \alpha+1], \alpha \in \mathbb{Z}$, $\phi$ is a polynomial of degree $\leq 2 d+1$ and

$$
\phi(\alpha)=w^{(0)}(\alpha), \quad \phi^{\prime}(\alpha)=w^{(1)}(\alpha), \quad \ldots, \quad \phi^{(d)}(\alpha)=w^{(d)}(\alpha), \quad \alpha \in \mathbb{Z} .
$$

Figure 1 is about the degree $d=2$. Three piecewise polynomials $\phi_{0}, \phi_{1}, \phi_{2}$ are considered for three Hermite problems:

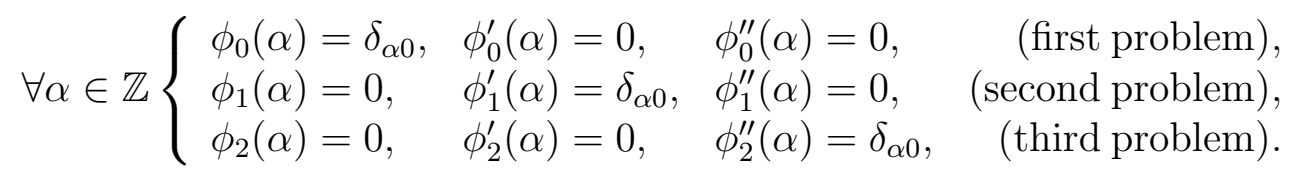



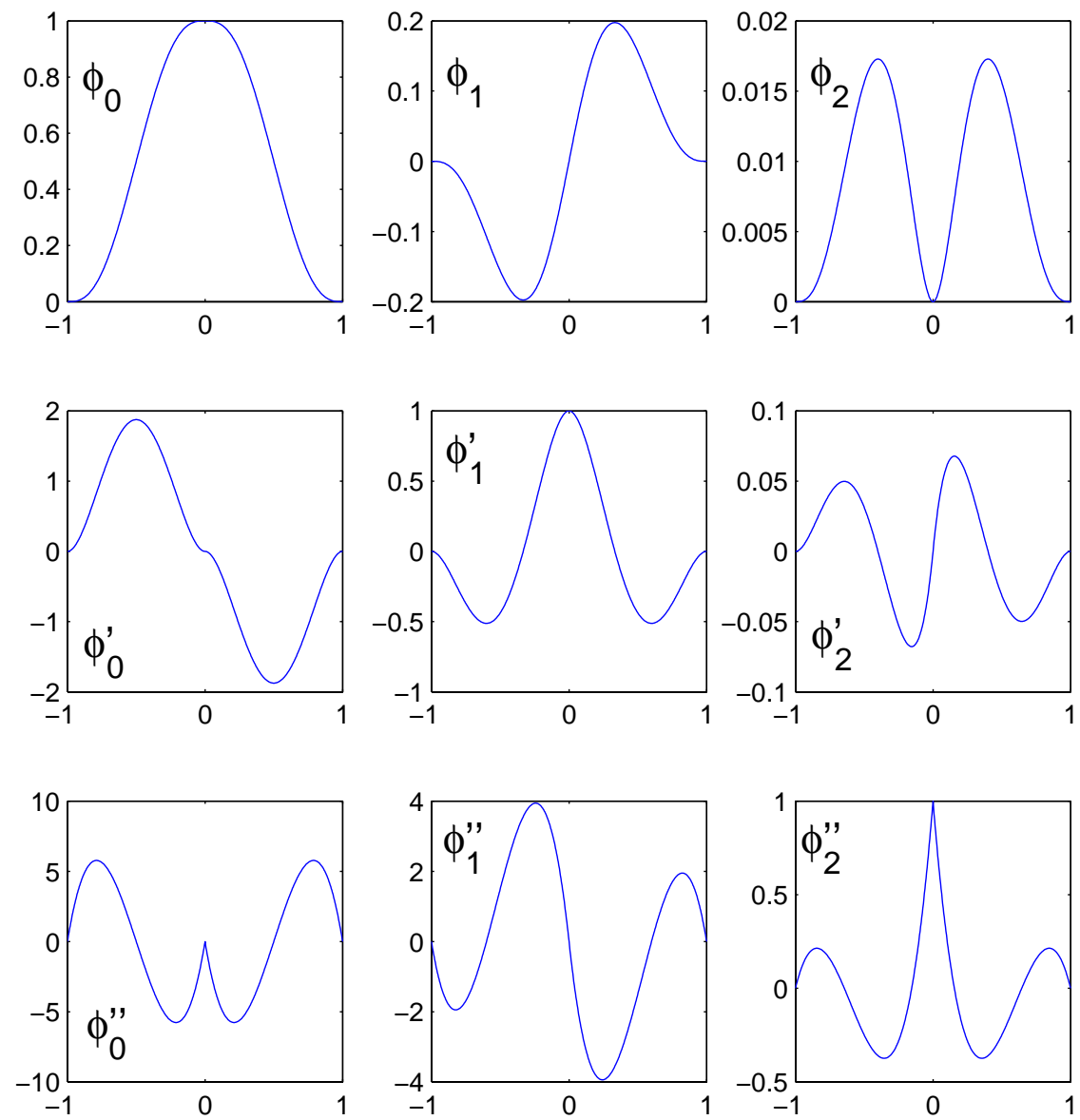

Figure 1: Basic Hermite interpolation functions and their derivatives of order 1 and 2 in the Hermite interpolation scheme of degree 2

Figure 1 provides the graphs of these functions with their two first derivatives.

Let us come back to the general Hermite problem (30). By sampling $\phi$ and its derivatives, we define a sequence of functions $f_{n}: \mathbb{Z} \rightarrow \mathbb{R}^{d+1}$,

$$
f_{n}(\alpha)=\left(\phi\left(\alpha / 2^{n}\right), \phi^{\prime}\left(\alpha / 2^{n}\right), \ldots, \phi^{(d)}\left(\alpha / 2^{n}\right)\right)^{T} .
$$

In (28), we set $a=\alpha / 2^{n}, b=(\alpha+1) / 2^{n}, y=f_{n}(\alpha), z=f_{n}(\alpha+1)$ and we obtain two equations by choosing $t=0$ then $t=1 / 2$ :

$$
\begin{aligned}
D^{n} f_{n+1}(2 \alpha) & =D^{n} f_{n}(\alpha), \\
D^{n} f_{n+1}(2 \alpha+1) & =U(1 / 2) D^{n} f_{n}(\alpha)+V(1 / 2) D^{n} f_{n}(\alpha+1) .
\end{aligned}
$$

If we multiply each left side of these equations by $D$, we get a Hermite subdivision scheme of degree $d$. The nonzero matrices of its mask are $A(0)=D, A(1)=D U(1 / 2)$, 
$A(-1)=D V(1 / 2)$ and we obtain

$$
D^{n+1} f_{n+1}(\alpha)=\sum_{\beta} A(\alpha-2 \beta) D^{n} f_{n}(\beta)
$$

We call it the Hermite polynomial interpolation scheme of degree $d, H I S^{d}$. It is an interpolatory scheme. Obviously the limit function of the refinements $f_{n}$ is $\phi$.

For $d=1,2,3$, the respective values of $A(1)=D U(1 / 2)$ are

$$
\frac{1}{8}\left(\begin{array}{rr}
4 & 1 \\
-6 & -1
\end{array}\right), \frac{1}{64}\left(\begin{array}{rrr}
32 & 10 & 1 \\
-60 & -14 & -1 \\
0 & -24 & -4
\end{array}\right), \frac{1}{768}\left(\begin{array}{rrrr}
384 & 132 & 18 & 1 \\
-840 & -228 & -24 & -1 \\
0 & -360 & -84 & -6 \\
2520 & 5040 & 360 & 18
\end{array}\right)
$$

The $i j$-entry of $A(-1)=D V(1 / 2)$ is $(-1)^{i+j}$ times the corresponding entry of $A(1)$ as it can be seen by (29).

As already said, the scheme $H I S^{d}$ is $C^{d}$, but this is also implied by Theorem 12 as we will see. Before this, we verify that the spectral condition is satisfied.

Lemma 13 Let $\{A(\alpha)\}$ be the mask of $H I S^{d}$ with $d>0$, then for $k=0,1, \ldots, 2 d+1$, the vector function $v_{k}(\alpha)=\left(p_{k}(\alpha), p_{k}^{\prime}(\alpha), \ldots, p_{k}^{(d)}(\alpha)\right)^{T}$ where $p_{k}(x)=x^{k}$ is an eigenvector of the supermatrix $H=(A(\alpha-2 \beta))_{\alpha, \beta \in \mathbb{Z}}$ with the eigenvalue $1 / 2^{k}$.

Proof: Let $p$ be a polynomial of degree less than or equal to $2 d+1$. If we sample $p$ and its derivatives on $2 d+1$ data, then the Hermite interpolating polynomial is $p$ again by unicity. We set $v(\alpha)=\left(p(\alpha), p^{\prime}(\alpha), \ldots, p^{(d)}(\alpha)\right)^{T}, v$ is a vector function from $\mathbb{Z}$ to $\mathbb{R}^{(d+1)}$. Let $V=(v(\alpha))_{\alpha \in \mathbb{Z}}$. If we define $W=(w(\alpha))_{\alpha \in \mathbb{Z}}=H V$ then

$$
w(\alpha)=\sum_{\beta \in \mathbb{Z}} A(\alpha-2 \beta) v(\beta) .
$$

From (31), we deduce that $w(\alpha)=\left(q(\alpha), q^{\prime}(\alpha), \ldots, q^{(d)}(\alpha)\right)^{T}$ where $q(x)=p(x / 2)$. For $k \in\{0,1, \ldots, 2 d+1\}$, if $p(x)=x^{k}$ then $q=x^{k} / 2^{k}$ and the corresponding vector $V_{k}=\left(v_{k}(\alpha)\right)_{\alpha \in \mathbb{Z}}$ is an eigenvector of $H$ with the eigenvalue $1 / 2^{k}$.

From Lemma 13 and Theorem 10, we can associate to $H I S^{d}$ the corresponding Taylor subdivision scheme $\mathcal{S}_{d}$. The mask of the subdivision scheme $\mathcal{S}_{d}$ is $B$ and can be computed by Theorem 10. From $B$, we get the subdivision matrix $S$. Since $\mathcal{S}_{d}$ is affine, we define the subdivision scheme $\Delta \mathcal{S}_{d}$ of the finite differences whose subdivision matrix is $T$. From Theorem 12, a criterion for the $C^{d}$ convergence of $H P P d$ is that there exists an integer $n$ for which $\left\|T^{n}\right\|_{\infty}<1$. We apply this criterion for the first schemes $H I S^{1}, H I S^{2}$ and $H I S^{3}$. 
For the scheme $H I S^{1}$, the subdivision matrices $S=(s(\alpha, \beta))$ and $T=(t(\alpha, \beta))$ are both periodic $s(\alpha+4, \beta+2)=s(\alpha, \beta)$ and $t(\alpha+4, \beta+2)=t(\alpha, \beta)$ for $\alpha, \beta \in \mathbb{Z}$. The respective matrices $(s(\alpha, \beta))$ and $(t(\alpha, \beta))$ for $\alpha=0,1,2,3$ and $\beta=0,1,2$ are

$$
\frac{1}{4}\left(\begin{array}{ccc}
4 & 0 & 0 \\
1 & 4 & -1 \\
-1 & 6 & -1 \\
-1 & 4 & 1
\end{array}\right), \quad \frac{1}{4}\left(\begin{array}{ccc}
3 & -1 & 0 \\
2 & 0 & 0 \\
0 & 2 & 0 \\
-1 & 3 & 0
\end{array}\right) .
$$

The entries $s(\alpha, \beta), t(\alpha, \beta)$ are 0 if $\alpha=[0,3]$ and $\beta \notin[0,2]$. With this information, $S$ and $T$ are entirely known. After computations, we obtain $\|T\|_{\infty}=1$ and $\left\|T^{2}\right\|_{\infty}=$ $5 / 8$. Since $\left\|T^{2}\right\|_{\infty}<1, H I S^{1}$ is $C^{1}$.

For the scheme $H I S^{2}$, the subdivision matrices $S$ and $T$ are both periodic $s(\alpha+$ $6, \beta+3)=s(\alpha, \beta)$ and $t(\alpha+6, \beta+3)=t(\alpha, \beta)$ for $\alpha, \beta \in \mathbb{Z}$. The respective matrices $(s(\alpha, \beta))$ and $(t(\alpha, \beta))$ for $\alpha \in[0,5]$ and $\beta \in[0,4]$ are

$$
\frac{1}{16}\left(\begin{array}{cccc}
16 & 0 & 0 & 0 \\
-1 & -14 & 30 & 1 \\
2 & -20 & 32 & 2 \\
-4 & 24 & 0 & -4 \\
1 & 46 & -30 & -1 \\
0 & 48 & -28 & -4
\end{array}\right), \quad \frac{1}{16}\left(\begin{array}{cccc}
17 & 31 & 1 & 0 \\
-3 & 3 & 1 & 0 \\
6 & -38 & -6 & 0 \\
-5 & -27 & 3 & 0 \\
1 & -1 & -3 & 0 \\
0 & 48 & 20 & 0
\end{array}\right) .
$$

The entries $s(\alpha, \beta), t(\alpha, \beta)$ are zero if $\alpha=[0,5]$ and $\beta \notin[0,4]$. The five first norms $\left\|T^{n}\right\|_{\infty}, n=1, \ldots, 5$, are $4.25,4.75,3.2422,1.8643,0.9962$. Since $\left\|T^{5}\right\|_{\infty}<1$, the Hermite scheme $H I S^{2}$ is $C^{2}$.

In a similar way, it is possible to verify that the Hermite scheme $H I S^{3}$ is $C^{3}$ by checking that $\left\|T^{7}\right\|_{\infty}=0.7486<1$ for the corresponding subdivision matrix $T$.

\subsection{Hermite corner cutting: $H C C^{d}$}

For every integer $d \geq 0$, we define another Hermite subdivision scheme of degree $d$. Let us denote $f_{0}: \mathbb{Z} \rightarrow \mathbb{R}^{d+1}$ a given family of control vectors. For $n=0,1,2, \ldots$, we will define recursively $f_{n+1}$ from $f_{n}$ by considering the unique piecewise polynomial $\phi_{n}: \mathbb{R} \rightarrow \mathbb{R}$ such that for every $\alpha \in \mathbb{Z}$

$$
\phi_{n}\left(\alpha / 2^{n}\right)=f_{n}^{(0)}(\alpha), \quad \phi_{n}^{\prime}\left(\alpha / 2^{n}\right)=f_{n}^{(1)}(\alpha), \quad \ldots, \quad \phi_{n}^{(d)}\left(\alpha / 2^{n}\right)=f_{n}^{(d)}(\alpha)
$$

and $\phi_{n}$ is a polynomial of degree $\leq 2 d+1$ in $\left[\alpha / 2^{n},(\alpha+1) / 2^{n}\right]$. The refined family of control vectors is set according to the rule

$$
f_{n+1}^{(i)}(\alpha)=\phi_{n}^{(i)}\left(\alpha / 2^{n+1}+1 / 2^{n+2}\right), \quad i=0, \ldots, d, \quad \alpha \in \mathbb{Z} .
$$



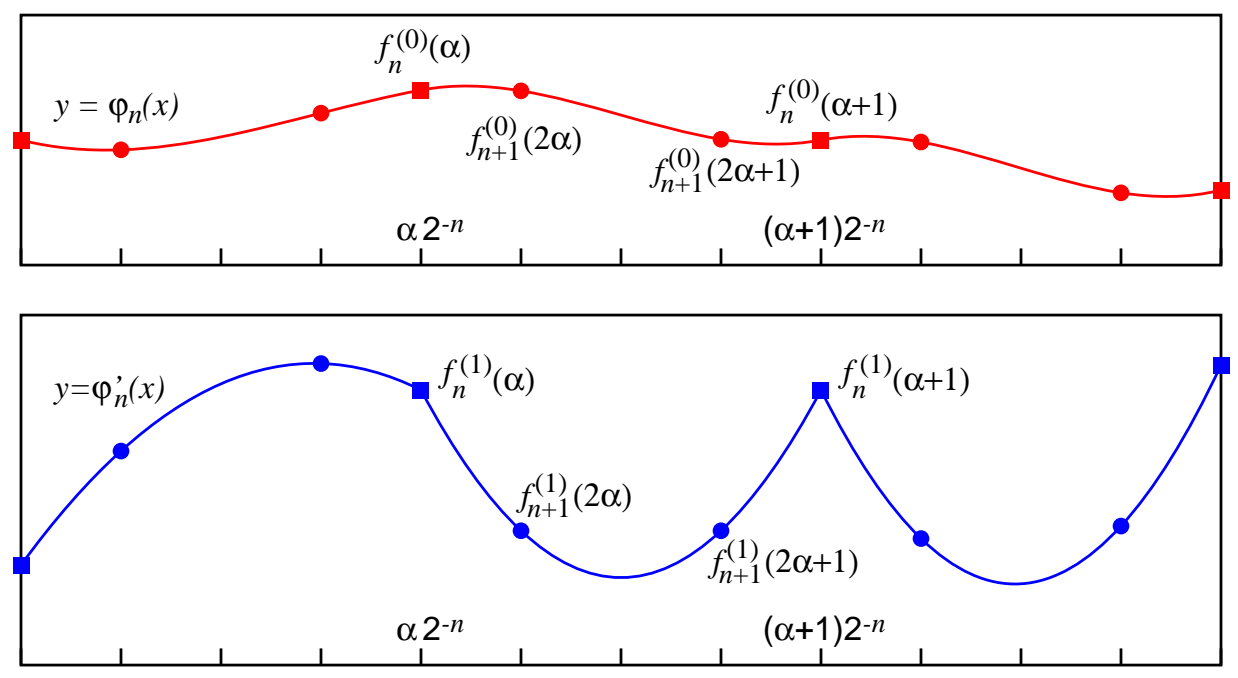

Figure 2: Hermite corner cutting of degree one

In Figure 2, we illustrate the refinement $f_{n+1}$ of $f_{n}$ according to (32) with $d=1$. Small black squares are related to $f_{n}$ and dots, to $f_{n+1}$.

In (28), we set $a=\alpha / 2^{n}, b=(\alpha+1) / 2^{n}, y=f_{n}(\alpha), z=f_{n}(\alpha+1)$ and we obtain two equations by choosing $t=1 / 4$ and $t=3 / 4$ :

$$
\begin{aligned}
D^{n} f_{n+1}(2 \alpha) & =U(1 / 4) D^{n} f_{n}(\alpha)+V(1 / 4) D^{n} f_{n}(\alpha+1), \\
D^{n} f_{n+1}(2 \alpha+1) & =U(3 / 4) D^{n} f_{n}(\alpha)+V(3 / 4) D^{n} f_{n}(\alpha+1) .
\end{aligned}
$$

If we multiply each left side of these equations by $D$, we get a Hermite subdivision scheme of degree $d$. The nonzero matrices of its mask are $A(0)=D U(1 / 4), A(-2)=$ $D V(1 / 4), A(1)=D U(3 / 4), A(-1)=D V(3 / 4)$ and we obtain

$$
D^{n+1} f_{n+1}(\alpha)=\sum_{\beta} A(\alpha-2 \beta) D^{n} f_{n}(\beta)
$$

We call it the Hermite corner cutting scheme of degree $d, H C C^{d}$.

If $d=0$, the previous formula becomes

$$
\begin{aligned}
f_{n+1}(2 \alpha) & =3 / 4 f_{n}(\alpha)+1 / 4 f_{n}(\alpha+1) \\
f_{n+1}(2 \alpha+1) & =1 / 4 f_{n}(\alpha)+3 / 4 f_{n}(\alpha+1)
\end{aligned}
$$

In this case this scheme is precisely Chaikin's algorithm [2], which belongs to a class of algorithms described by G. de Rham [4]. The name of corner cutting has been given to the class of de Rham's algorithms. 
Let us look at the mask of $H C C^{1}$. The matrices $A(0)=D U(1 / 4), A(1)=$ $D U(3 / 4)$ respectively are

$$
\frac{1}{64}\left(\begin{array}{rr}
54 & 9 \\
-36 & 6
\end{array}\right), \quad \frac{1}{64}\left(\begin{array}{rr}
10 & 3 \\
-36 & -10
\end{array}\right) .
$$

The matrices $A(-2)=D V(1 / 4)$ and $A(-1)=D V(3 / 4)$ can be computed from (29). The $i j$-entry of $A(-1)$ is $(-1)^{i+j}$ times the corresponding entry of $A(1)$, the $i j$-entry of $A(-2)$ is $(-1)^{i+j}$ times the corresponding entry of $A(0)$.

In the case of $H C C^{2}$, the respective matrices $A(0)=D U(1 / 4)$ and $A(1)=$ $D U(3 / 4)$ of its mask are

$$
\frac{1}{2048}\left(\begin{array}{rrr}
1836 & 378 & 27 \\
-1080 & 324 & 54 \\
-2880 & -2016 & -144
\end{array}\right), \quad \frac{1}{2048}\left(\begin{array}{rrr}
212 & 78 & 9 \\
-1080 & -380 & -42 \\
3880 & 864 & 80
\end{array}\right) .
$$

A computation by symmetry provides $A(-1)$ and $A(-2)$.

The fact that the scheme $H C C^{d}$ satisfies the spectral condition comes from the following lemma.

Lemma 14 Let $\{A(\alpha)\}$ be the mask of the Hermite corner cutting of degree $d>0$, then for $k=0,1, \ldots, 2 d+1$, the vector function $v_{k}(\alpha)=\left(p_{k}(\alpha), p_{k}^{\prime}(\alpha), \ldots, p_{k}^{(d)}(\alpha)\right)^{T}$ where $p_{k}(x)=(x-1 / 2)^{k}$ is an eigenvector of the supermatrix $H=(A(\alpha-2 \beta))_{\alpha, \beta \in \mathbb{Z}}$ with the eigenvalue $1 / 2^{k}$.

Proof: We use a similar proof to the one of Lemma 13. With the same notations, from $v(\alpha)=\left(p(\alpha), p^{\prime}(\alpha), \ldots, p^{(d)}(\alpha)\right)^{T}$, with (32), we obtain that $w(\alpha)=$ $\left(q(\alpha), q^{\prime}(\alpha), \ldots, q^{(d)}(\alpha)\right)^{T}$ where $q(x)=p(x / 2+1 / 4)$. For $k \in\{0,1, \ldots, 2 d+1\}$, if $p=(x-1 / 2)^{k}$, then $q=(x-1 / 2)^{k} / 2^{k}$ and $V_{k}$ is an eigenvector of $H$ with the eigenvalue $1 / 2^{k}$.

From Lemma 13 and Theorem 10, we can associate with $H C C^{d}$ the corresponding Taylor subdivision scheme $\mathcal{S}_{d}$. We consider the subdivision scheme $\Delta \mathcal{S}_{d}$ of the finite differences whose subdivision matrix is $T$. From Theorem $12, H C C^{d}$ is $C^{d}$ if there exists an integer $n$ for which $\left\|T^{n}\right\|_{\infty}<1$. We apply this criterion for the first schemes $H C C^{1}, H C C^{2}$ and $H C C^{3}$.

For the scheme $H C C^{1}$, the subdivision matrices $S=(s(\alpha, \beta))$ and $T=(t(\alpha, \beta))$ are both periodic $s(\alpha+4, \beta+2)=s(\alpha, \beta)$ and $t(\alpha+4, \beta+2)=t(\alpha, \beta)$ for $\alpha, \beta \in \mathbb{Z}$. The respective matrices $(s(\alpha, \beta))$ and $(t(\alpha, \beta))$ for $\alpha=0,1,2,3$ and $\beta=0,1,2,3,4$ are

$$
\frac{1}{32}\left(\begin{array}{ccccc}
6 & 36 & -10 & 0 & 0 \\
-6 & 44 & -6 & 0 & 0 \\
-10 & 36 & 6 & 0 & 0 \\
-3 & 10 & 18 & 10 & -3
\end{array}\right), \quad \frac{1}{32}\left(\begin{array}{cccc}
12 & 4 & 0 & 0 \\
4 & 12 & 0 & 0 \\
-7 & 19 & 7 & -3 \\
-3 & 7 & 19 & -7
\end{array}\right) .
$$


The entries $s(\alpha, \beta), t(\alpha, \beta)$ are 0 if $\alpha=[0,3]$ and $\beta \notin[0,4]$. With this information, $S$ and $T$ are entirely known. After computations, we obtain $\|T\|_{\infty}=9 / 8$ and $\left\|T^{2}\right\|_{\infty}=$ 5/8. Since $\left\|T^{2}\right\|_{\infty}<1, H C C^{1}$ is $C^{1}$.

For Figure 3, let $j \in\{0,1,2\}$, we consider the initial state of $H C C^{2}, f_{0}(\alpha)=$ $\delta_{\alpha 0}\left(\delta_{j 0}, \delta_{j 1}, \delta_{j 2}\right)^{T}$ for $\alpha \in \mathbb{Z}$. The corresponding sequence of refinements $f_{n}$ converges to a vector function: $\left(\phi_{j}, \phi_{j}^{\prime}, \phi^{\prime \prime}\right)^{T}$. Figure 3 provides the graphs of these functions. The support of each function $\phi_{j}$ is $[-1,2]$.
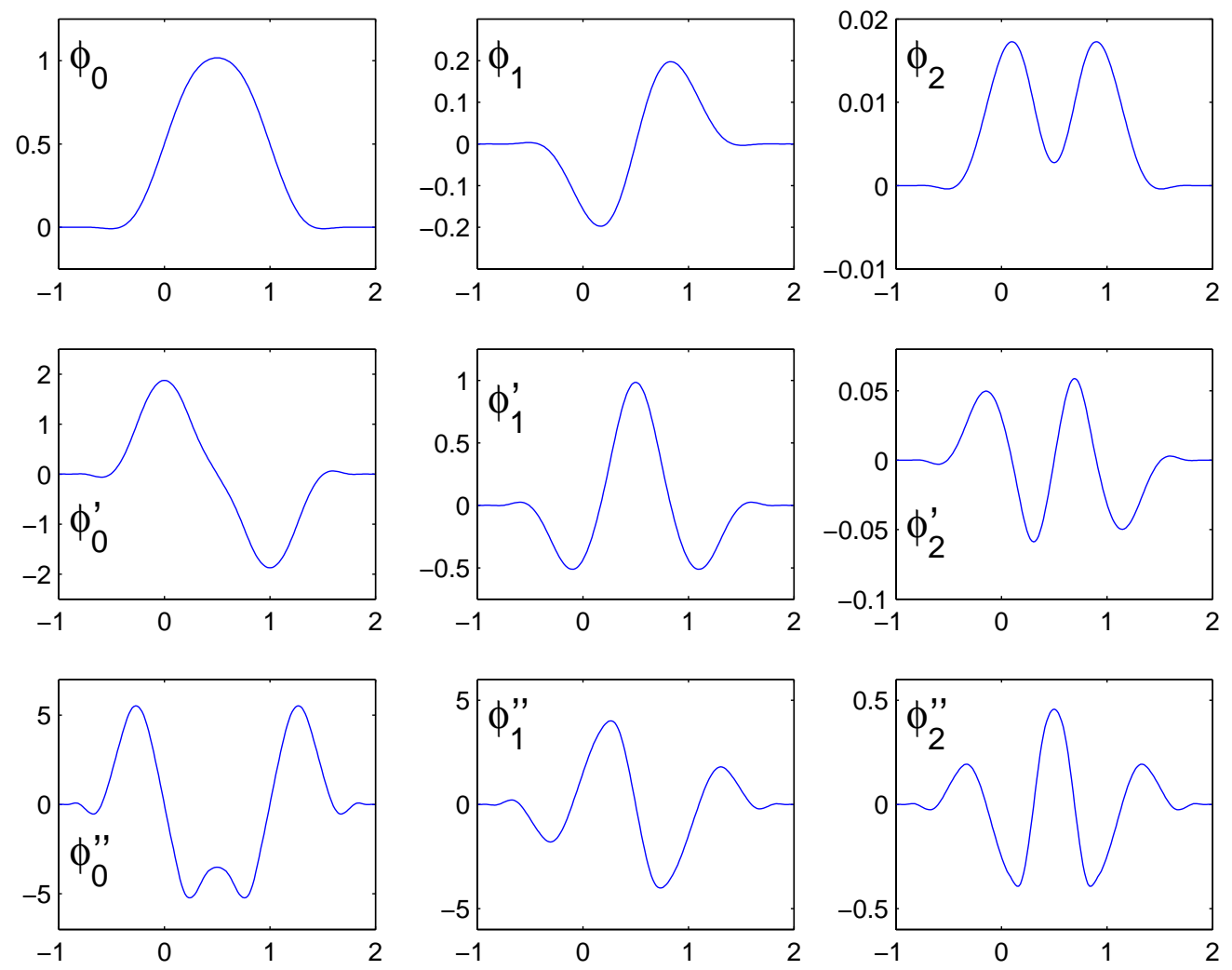

Figure 3: Basic limit functions and their derivatives of order 1 and 2 in the Hermite corner cutting of degree 2

For the scheme $H C C^{2}$, the subdivision matrix $T$ is periodic $t(\alpha+6, \beta+3)=t(\alpha, \beta)$ for $\alpha, \beta \in \mathbb{Z}$. The matrix $(t(\alpha, \beta))$ for $\alpha \in[0,5]$ and $\beta \in[0,5]$ is

$$
\frac{1}{256}\left(\begin{array}{cccccc}
-24 & -808 & -88 & 0 & 0 & 0 \\
24 & 296 & 24 & 0 & 0 & 0 \\
-112 & -1040 & -48 & 0 & 0 & 0 \\
19 & 677 & 227 & 101 & 291 & 21 \\
-12 & -228 & -64 & -64 & -176 & -12 \\
33 & 599 & 165 & 291 & 645 & 31
\end{array}\right) .
$$

The entries $t(\alpha, \beta)$ are zero if $\alpha=[0,5]$ and $\beta \notin[0,5]$. The five first norms $\left\|T^{n}\right\|_{\infty}$, 
$n=1, \ldots, 5$, are $6.8906,5.2480,2.8572,1.4597,0.7342$. Since $\left\|T^{5}\right\|_{\infty}<1, H C C^{2}$ is $C^{2}$.

In a similar way, it is possible to verify that the Hermite corner cutting of degree 3 is $C^{3}$ by checking that $\left\|T^{7}\right\|_{\infty}=0.6840<1$ for the corresponding subdivision matrix $T$.

\section{Conclusion}

Our goal was to get a criterion for the $C^{d}$ convergence of a Hermite subdivision scheme $\mathcal{H}$ of degree $d>0$ in dimension 1 . Under the spectral condition, we transformed the Hermite subdivision scheme $\mathcal{H}$ into the Taylor subdivision scheme $\mathcal{S}$ and the $C^{d}$-convergence of $\mathcal{H}$ has been reduced to the $C^{0}$-convergence of $\mathcal{S}$ with equal components. This criterion has been applied to two families of Hermite subdivision schemes, the first being interpolatory, the second being the extension of corner cutting to Hermite subdivision schemes. Noninterpolatory schemes seem to be smoother than interpolatory ones.

\section{References}

[1] M. D. Buhmann and C. Micchelli, Using two-slanted matrices for subdivision. Proc. London Math. Soc. (3) 69 (1994), 428-448.

[2] G. Chaikin, An algorithm for high speed curve generation. Computer Graphics and Image Processing 3 (1974), 346-349.

[3] I. Daubechies, I. Guskov and W. Sweldens, Commutation for Irregular Subdivision. Constr. Approx. 17 (2001) 479-514.

[4] G. de Rham, Sur une courbe plane. J. Math. Pures Appli. 35 (1956), 25-42.

[5] S. Dubuc and J.-L. Merrien, Convergent vector and Hermite subdivision schemes. Constr. Approx. 23 ( 2006) 1-22.

[6] S. Dubuc, Scalar and Hermite subdivision schemes, Appl. Comp. Harm. Anal. 21 (2006), 376-394.

[7] N. Dyn, D. Levin, Analysis of Hermite-type subdivision schemes. In C. K. Chui and L. L. Schumaker Eds. Approximation Theory VIII. Vol 2: Wavelets and Multilevel Approximation (College Station, TX, 1995). World Sci., River Edge, NJ, 1995, pp. 117-124. 
[8] N. Dyn, D. Levin, Analysis of Hermite-interpolatory subdivision schemes. In Spline Functions and the Theory of Wavelets, S. Dubuc et G. Deslauriers, Ed. Amer. Math. Soc., Providence R. I., 1999, pp. 105-113.

[9] B. Han, Approximation properties and construction of Hermite interpolants and biorthogonal multiwavelets, J. Approx. Theory 110 (2001), 18-53.

[10] S. Lang, Linear Algebra. Reprint of the third edition. Springer-Verlag, New York, 1989.

[11] D. Levin, Using Laurent polynomial representation for the analysis of nonuniform binary subdivision schemes. Adv. Comput. Math. 11 (1999), 41-54.

[12] V. Maxim and M.-L. Mazure, Subdivision schemes and irregular grids. Numer. Algorithms 35 (2004), 1-28.

[13] J.-L. Merrien, A family of Hermite interpolants by bisection algorithms, Numer. Algorithms 2 (1992), 187-200.

[14] J.-L. Merrien, Interpolants d'Hermite $C^{2}$ obtenus par subdivision, M2AN Math. Model. Numer. Anal. 33 (1999), no. 1, 55-65.

[15] N. Obreshkov, On the mechanical quadratures. J. Bulg. Acad. Sci. and Arts, 65 (1942) 191-289.

[16] B. Sendov, A. Andrew, Approximation and Interpolation Theory. In P. G. Ciarlet, J. L. Lions Eds, Handbook of Numerical Analysis III. North-Holland, 1994, 223-462.

[17] T. P.-Y. Yu, On the regularity analysis of interpolatory Hermite subdivision schemes, J. Math. Anal. Appl. 302 (2005), 201-216.

[18] D.-X. Zhou, Multiple refinable Hermite interpolants. J. Approx. Theory 102 (2000), 46-71. 